\title{
Systematic Review \\ The Role of miRNAs in the Regulation of Endometrial Cancer Invasiveness and Metastasis-A Systematic Review
}

\author{
Klaudia Klicka ${ }^{1,2,+}+$, Tomasz M. Grzywa ${ }^{1,2,3,+}{ }^{\infty}$, Alicja Klinke ${ }^{1}$, Aleksandra Mielniczuk ${ }^{1}$ \\ and Paweł K. Włodarski ${ }^{1, *(1)}$ \\ 1 Department of Methodology, Medical University of Warsaw, 02-091 Warsaw, Poland; \\ klaudia.klicka@wum.edu.pl (K.K.); tomasz.grzywa@wum.edu.pl (T.M.G.); \\ s073727@student.wum.edu.pl (A.K.); s073849@student.wum.edu.pl (A.M.) \\ 2 Doctoral School, Medical University of Warsaw, 02-091 Warsaw, Poland \\ 3 Department of Immunology, Medical University of Warsaw, 02-097 Warsaw, Poland \\ * Correspondence: pawel.wlodarski@wum.edu.pl \\ + These authors contributed equally to this work.
}

check for updates

Citation: Klicka, K.; Grzywa, T.M.; Klinke, A.; Mielniczuk, A.; Włodarski, P.K. The Role of miRNAs in the Regulation of Endometrial Cancer Invasiveness and Metastasis-A Systematic Review. Cancers 2021, 13, 3393. https://doi.org/10.3390/ cancers13143393

Academic Editors: Miguel Abal and Laura Muinelo-Romay

Received: 5 May 2021

Accepted: 2 July 2021

Published: 6 July 2021

Publisher's Note: MDPI stays neutral with regard to jurisdictional claims in published maps and institutional affiliations.

Copyright: (c) 2021 by the authors. Licensee MDPI, Basel, Switzerland. This article is an open access article distributed under the terms and conditions of the Creative Commons Attribution (CC BY) license (https:/ / creativecommons.org/licenses/by/ $4.0 /)$.
Simple Summary: Endometrial cancer (EC) is one of the most frequent cancers with increasing annual death rates. Therefore, it is of great clinical importance to understand the mechanisms of endometrial cancer invasiveness and metastasis. MiRNAs are small single-stranded RNAs that regulate gene expression. They were discovered to play a role in all steps of cancer development. This study aimed at conducting a systematic review of the role of miRNAs in endometrial cancer invasiveness and metastasis. The collected data demonstrate that miRNAs regulate EC invasiveness and metastasis by different targets. MiRNAs seem to be potential candidates for diagnostic and prognostic biomarkers, as well as possible therapeutic targets.

Abstract: Endometrial cancer (EC) is the most common genital cancer in women with increasing death rates. MiRNAs are short non-coding RNAs that regulate gene expression on the post-transcriptional levels. Multiple studies demonstrated a fundamental role of miRNAs in the regulation of carcinogenesis. This systematic review is a comprehensive overview of the role of miRNAs in the regulation of cancer cell invasiveness and metastasis in EC. The literature was searched for studies investigating the role of miRNAs in the regulation of invasiveness and metastasis in EC. We explored PubMed, Embase, and Scopus using the following keywords: miRNA, metastasis, invasiveness, endometrial cancer. Data were collected from 163 articles that described the expression and role of 106 miRNAs in the regulation of EC invasiveness and metastasis out of which 63 were tumor suppressor miRNAs, and 38 were oncomiRNAs. Five miRNAs had a discordant role in different studies. Moreover, we identified 66 miRNAs whose expression in tumor tissue or concentration in serum correlated with at least one clinical parameter. These findings suggest a crucial role of miRNAs in the regulation of EC invasiveness and metastasis and present them as potential prognostic factors for patients with EC.

Keywords: endometrial cancer; miRNAs; metastasis; invasiveness; migration; biomarker

\section{Introduction}

Endometrial cancer (EC) accounts for the most frequent cancers with growing incidence rates [1,2]. The outcome depends on the progression of the disease and applied treatment [3,4]. That makes effective management of EC risk factors, early diagnosis, and effective therapy strategies of EC the clinically important challenges. There are two most common EC staging classifications, TNM and The International Federation of Gynecology and Obstetrics (FIGO) that differentiate endometrial cancer tumors according to the depth of invasion and presence of metastases (Figure 1) [5]. Furthermore, there is a FIGO grading based on the level of glandular differentiation. A higher grade is associated with a non-glandular, non-squamous growth [6]. 


\section{Endometrial cancer staging and histological grading}
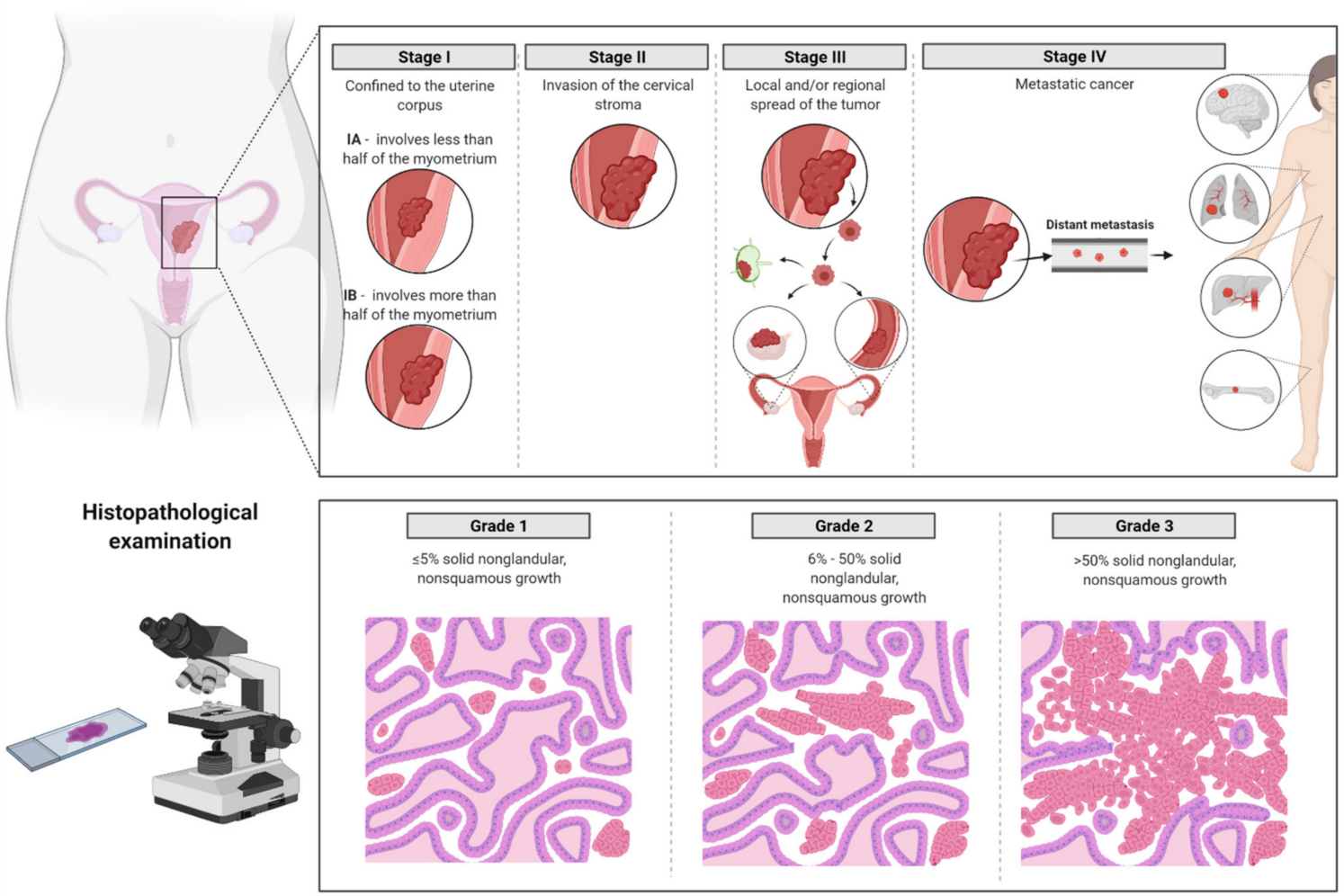

Figure 1. Federation of Gynecology and Obstetrics (FIGO) staging and histological grading of endometrial cancer. Figure was created using Biorender.com.

MiRNAs are small non-coding single-stranded molecules that regulate all hallmarks of cancer by influencing gene expression post-transcriptionally. The formation of miRNAs begins in the nucleus where polymerase II (Pol II) transcribes pri-miRNA. The pri-miRNA is cropped by the DROSHA complex and exported by exportin 5 to the cytoplasm where mature single-stranded miRNAs arise with the participation of DICER and Argonaute 2 (AGO2) $[7,8]$. During carcinogenesis, the profile of miRNAs expression undergoes a substantial dysregulation $[9,10]$. It is a result of multiple changes, including amplification and deletion of miRNA genes or dysregulation of epigenetics [9]. Moreover, miRNAs expression is dysregulated in cancer as an effect of defects in miRNA biogenesis machinery, including DICER and DROSHA [11].

MiRNAs take part in all steps of tumor cell invasiveness and metastasis including migration, local invasion, epithelial-mesenchymal transition (EMT), and systemic circulation [12]. The same miRNAs may play opposite roles in different tumors, promoting tumor growth (oncomiRNAs) or acting as tumor suppressor miRNAs [13]. By targeting $3^{\prime}$ untranslated region (UTR) of multiple mRNAs they regulate all hallmarks of cancer defined by Hanahan and Weinberg, including proliferation, invasion, angiogenesis, as well as they influence cancer cells chemoresistance [12,14-17].

This systematic review aims to highlight the complex role of miRNAs in regulating endometrial cancer invasion and metastasis. We focus on the aberrant expression of different miRNAs in endometrial cancer tissues and cell lines and their role in the regulation of tumor invasion, metastasis, and patients' outcomes.

\section{Materials and Methods}

\subsection{Search Strategy}

The Preferred Reporting Items for Systematic Reviews and Meta-Analyses (PRISMA) were used to ensure reporting transparency (Figure 2). Two reviewers (KK and TMG) collected, screened, and performed an independent assessment of the quality of the studies. 
Discrepancies were discussed and resolved by consensus. The literature systematic search was undertaken using MEDLINE (PubMed), Embase, and Scopus (9 March 2021) with the terms ('microRNA' OR 'miRNA') AND ('metastasis' OR 'invasiveness') AND ('endometrial cancer' OR 'endometrial carcinoma'). Duplicates were deleted. The references of the found studies were reviewed to find other records.

\section{Search strategy and study selection}

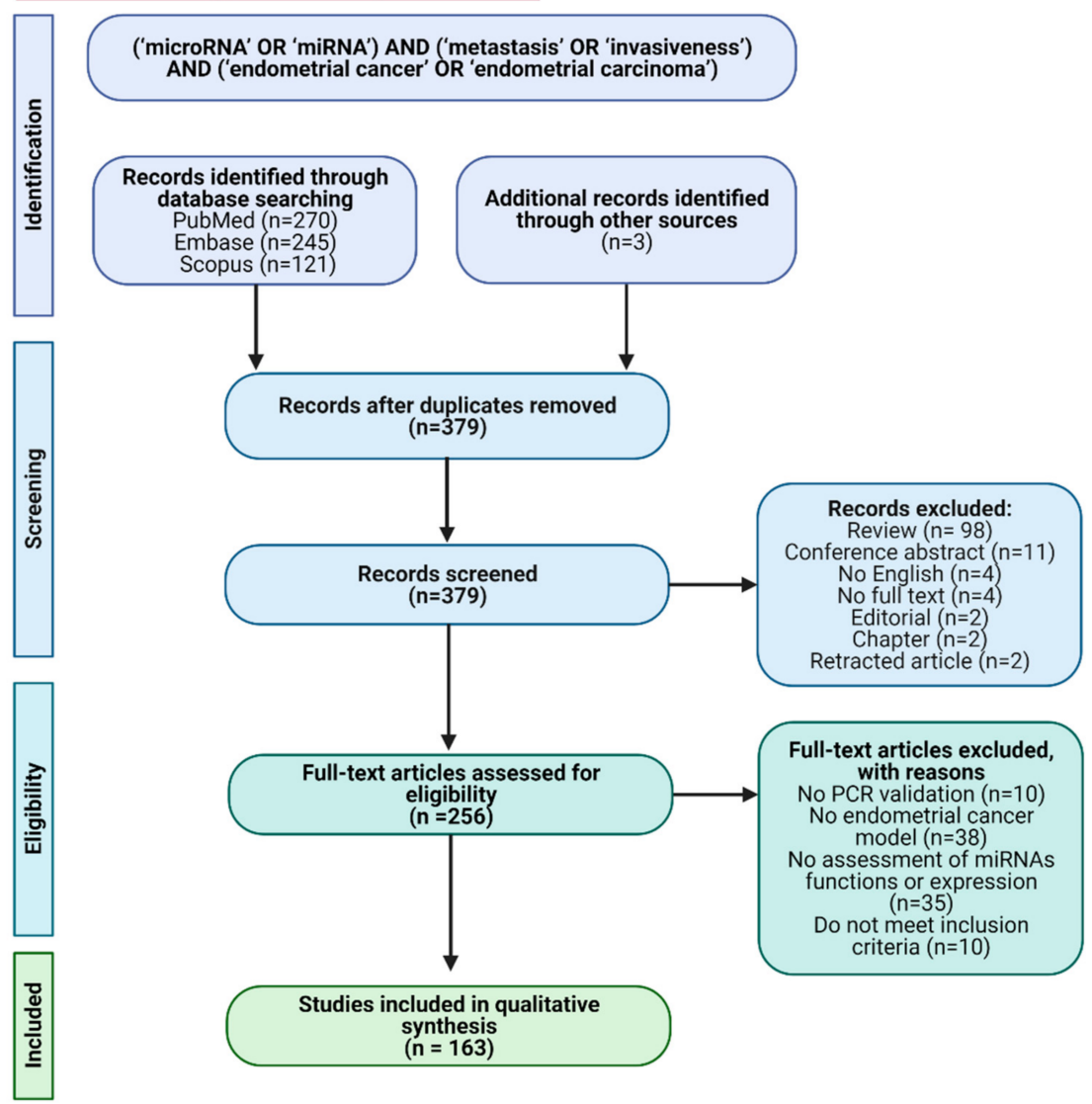

Figure 2. PRISMA flow-chart diagram of study selection. $\mathrm{n}=$ number of articles. Figure was created using Biorender.com.

\subsection{Inclusion and Exclusion Criteria}

The articles were included if they answered the PICO question (Table 1). The studies with the assessment of miRNA expression levels and the role of miRNAs in endometrial cancer cell invasion, migration, or outcome were allowed. We assessed only original articles written in English with full-text available. Review articles, chapters, conference abstracts, and retracted articles were excluded. Full texts were assessed for eligibility. We excluded articles that did not meet inclusion criteria, articles with no assessment of miRNAs function or expression in the endometrial cancer model, and no validation with RT-qPCR (real-time quantitative PCR) of miRNA expression.

Table 1. PICO question form.

\begin{tabular}{cc}
\hline Domain & Inclusion Criteria \\
\hline Patients $(\mathrm{P})$ & Patients with endometrial cancer or endometrial cancer cells \\
Interventions (I) & Differentially expressed miRNAs \\
Comparators $(\mathrm{C})$ & Non-neoplastic endometrium or cells \\
Outcomes $(\mathrm{O})$ & Tumor invasiveness or metastasis \\
\hline
\end{tabular}




\subsection{Data Extraction}

The data extracted from the revised full-texts were the levels of expression of miRNAs in human tissues, human serum, and cell lines, the role of miRNAs in the regulation of migration, invasion, and EMT in vitro, as well as tumor growth and metastasis in vivo, the outcomes of patients, the overall survival (OS), disease-free survival (DFS), progressionfree survival (PFS), FIGO stage, histological grade, myometrial invasion, and lymph node metastases.

\section{Results}

The detailed results of the literature search are presented in Figure 2. The search strategy provided a total of 270 records in the PubMed database, 245 in the Embase, 121 in the Scopus database, and three records identified through other sources. In total, 379 records remained after the removal of duplicated articles. Out of 379,123 records were excluded with reasons (no validation by RT-qPCR, no EC model, no assessment of miRNA functions or expression, or records that do not meet inclusion criteria). A total of 256 fulltext articles were assessed for eligibility. Finally, data were collected from 163 articles demonstrating the role of miRNA in the regulation of EC invasiveness or metastasis.

\subsection{MiRNAs Dysregulation in Endometrial Cancer}

Our search identified 115 articles that revealed 106 miRNAs regulating invasiveness and metastasis that are dysregulated in EC, which was confirmed by RT-qPCR (Table 2) [18-132]. In total, 33 miRNAs were confirmed to be dysregulated by at least two studies. Most of them (63) are tumor suppressors and are downregulated in EC, while 38 are oncomiRNAs upregulated in cancer tissue. Five miRNAs have discordant expression levels in different studies.

Table 2. Dysregulation of expression of invasiveness-associated miRNAs in endometrial cancer.

\begin{tabular}{|c|c|c|c|c|c|c|c|c|c|}
\hline MiRNA & $\begin{array}{l}\text { Human } \\
\text { Tissue }\end{array}$ & $\begin{array}{l}\text { Cell } \\
\text { Line }\end{array}$ & $\begin{array}{c}\text { Circulating } \\
\text { miRNA }\end{array}$ & Ref. & MiRNA & $\begin{array}{l}\text { Human } \\
\text { Tissue }\end{array}$ & $\begin{array}{l}\text { Cell } \\
\text { Line }\end{array}$ & $\begin{array}{l}\text { Circulating } \\
\text { miRNA }\end{array}$ & Ref. \\
\hline let-7b & $\downarrow$ & $\downarrow$ & $\mathrm{n} / \mathrm{d}$ & [41] & $\operatorname{miR}-10 b$ * & $\uparrow$ & $\mathrm{n} / \mathrm{d}$ & $\uparrow$ & {$[118,129]$} \\
\hline miR-1 & $\downarrow$ & $\downarrow$ & $\mathrm{n} / \mathrm{d}$ & {$[97]$} & miR-21 & & $\mathrm{n} / \mathrm{d}$ & $\mathrm{n} / \mathrm{d}$ & {$[57,75,107]$} \\
\hline $\operatorname{miR}-10 b$ * & $\downarrow$ & $\mathrm{n} / \mathrm{d}$ & $\mathrm{n} / \mathrm{d}$ & {$[38,78]$} & miR-27 & & $\mathrm{n} / \mathrm{d}$ & $\uparrow$ & [60] \\
\hline miR-20a-5p & $\downarrow$ & $\downarrow$ & $\mathrm{n} / \mathrm{d}$ & {$[40]$} & miR-34a * & & $\mathrm{n} / \mathrm{d}$ & $\mathrm{n} / \mathrm{d}$ & [61] \\
\hline miR-20b-5p & $\downarrow$ & $\downarrow$ & $\mathrm{n} / \mathrm{d}$ & [116] & miR-93 & & $\mathrm{n} / \mathrm{d}$ & $\mathrm{n} / \mathrm{d}$ & [27] \\
\hline miR-22 & $\downarrow$ & $\downarrow$ & $\mathrm{n} / \mathrm{d}$ & {$[77,121]$} & miR-93-5p & & $\uparrow$ & $\mathrm{n} / \mathrm{d}$ & {$[95,105]$} \\
\hline $\mathrm{miR}-23 \mathrm{a}$ & $\downarrow$ & $\mathrm{n} / \mathrm{d}$ & $\downarrow$ & {$[118,128]$} & miR-95 & & $\mathrm{n} / \mathrm{d}$ & & [110] \\
\hline miR-26a & $\mathrm{n} / \mathrm{d}$ & $\mathrm{n} / \mathrm{d}$ & $\downarrow$ & [42] & miR-99a & $\mathrm{n} / \mathrm{d}$ & $\mathrm{n} / \mathrm{d}$ & $\uparrow$ & [76] \\
\hline miR-29a-5p & $\downarrow$ & $\downarrow$ & $\mathrm{n} / \mathrm{d}$ & [44] & miR-100 & $\mathrm{n} / \mathrm{d}$ & $\mathrm{n} / \mathrm{d}$ & & [76] \\
\hline miR-29b & $\downarrow$ & $\mathrm{n} / \mathrm{d}$ & $\downarrow$ & {$[24,38,81]$} & miR-106a & & & $\mathrm{n} / \mathrm{d}$ & [73] \\
\hline miR-30c & $\downarrow$ & $\mathrm{n} / \mathrm{d}$ & $\mathrm{n} / \mathrm{d}$ & [96] & miR-107-5p & $\uparrow$ & $\uparrow$ & $\mathrm{n} / \mathrm{d}$ & [19] \\
\hline miR-34 & $\downarrow$ & $\mathrm{n} / \mathrm{d}$ & $\mathrm{n} / \mathrm{d}$ & [57] & miR-130b * & & $\mathrm{n} / \mathrm{d}$ & $\mathrm{n} / \mathrm{d}$ & [122] \\
\hline $\operatorname{miR}-34 a^{*}$ & $\downarrow$ & $\mathrm{n} / \mathrm{d}$ & $\mathrm{n} / \mathrm{d}$ & [34] & miR-141 & & $\mathrm{n} / \mathrm{d}$ & $\uparrow$ & {$[35,47,77]$} \\
\hline miR-34b & $\downarrow$ & $\mathrm{n} / \mathrm{d}$ & $\mathrm{n} / \mathrm{d}$ & {$[38,39,101]$} & miR-145 & $\uparrow$ & $\mathrm{n} / \mathrm{d}$ & $\mathrm{n} / \mathrm{d}$ & [21] \\
\hline $\mathrm{miR}-34 \mathrm{c}$ & $\downarrow$ & $\mathrm{n} / \mathrm{d}$ & $\mathrm{n} / \mathrm{d}$ & {$[43,123]$} & miR-146a & $\uparrow$ & $\mathrm{n} / \mathrm{d}$ & $\mathrm{n} / \mathrm{d}$ & [114] \\
\hline miR-99a & $\downarrow$ & $\mathrm{n} / \mathrm{d}$ & $\mathrm{n} / \mathrm{d}$ & {$[51,76]$} & miR-148b & $\uparrow$ & $\mathrm{n} / \mathrm{d}$ & $\mathrm{n} / \mathrm{d}$ & [57] \\
\hline miR-100 & $\downarrow$ & $\mathrm{n} / \mathrm{d}$ & $\mathrm{n} / \mathrm{d}$ & [76] & miR-181a & 1 & $\mathrm{n} / \mathrm{d}$ & $\mathrm{n} / \mathrm{d}$ & [37] \\
\hline miR-101 & $\downarrow$ & $\downarrow$ & $\mathrm{n} / \mathrm{d}$ & $\begin{array}{l}{[38,46,56,} \\
106,119]\end{array}$ & miR-182 & $\uparrow$ & $\mathrm{n} / \mathrm{d}$ & $\mathrm{n} / \mathrm{d}$ & {$[30,47,48,77]$} \\
\hline miR-124 & $\downarrow$ & $\mathrm{n} / \mathrm{d}$ & $\mathrm{n} / \mathrm{d}$ & {$[33,50,99]$} & miR-183 & $\uparrow$ & $\mathrm{n} / \mathrm{d}$ & $\mathrm{n} / \mathrm{d}$ & {$[30,48,62,77,92]$} \\
\hline miR-125 & $\downarrow$ & $\mathrm{n} / \mathrm{d}$ & $\mathrm{n} / \mathrm{d}$ & [57] & miR-192 & & $\mathrm{n} / \mathrm{d}$ & $\mathrm{n} / \mathrm{d}$ & [30] \\
\hline miR-125b & $\downarrow$ & $\mathrm{n} / \mathrm{d}$ & $\mathrm{n} / \mathrm{d}$ & {$[63,104]$} & miR-194 & $\uparrow$ & $\mathrm{n} / \mathrm{d}$ & $\mathrm{n} / \mathrm{d}$ & [30] \\
\hline miR-126 & $\downarrow$ & $\mathrm{n} / \mathrm{d}$ & $\mathrm{n} / \mathrm{d}$ & {$[109,120]$} & miR-200a & $\uparrow$ & $\mathrm{n} / \mathrm{d}$ & $\uparrow$ & {$[35,48,77,102]$} \\
\hline miR-130b * & $\downarrow$ & $\mathrm{n} / \mathrm{d}$ & $\mathrm{n} / \mathrm{d}$ & [32] & miR-200b & $\uparrow$ & $\mathrm{n} / \mathrm{d}$ & $\mathrm{n} / \mathrm{d}$ & {$[31,77,102]$} \\
\hline miR-133a & $\downarrow$ & $\downarrow$ & $\mathrm{n} / \mathrm{d}$ & {$[38,97]$} & miR-200c & $\uparrow$ & $\uparrow$ & $\mathrm{n} / \mathrm{d}$ & {$[26,47,48,53,77]$} \\
\hline $\operatorname{miR}-133 b$ & $\downarrow$ & $\mathrm{n} / \mathrm{d}$ & $\mathrm{n} / \mathrm{d}$ & [38] & miR-203 & $\uparrow$ & $\mathrm{n} / \mathrm{d}$ & $\uparrow$ & {$\left[\begin{array}{c}\text { [77] } \\
{[29303538,47}\end{array}\right.$} \\
\hline miR-136 & $\downarrow$ & $\mathrm{n} / \mathrm{d}$ & $\mathrm{n} / \mathrm{d}$ & [65] & $\operatorname{miR}-205^{*}$ & $\uparrow$ & $\mathrm{n} / \mathrm{d}$ & $\mathrm{n} / \mathrm{d}$ & $\begin{array}{c}48,59,68,77,78, \\
89,91]\end{array}$ \\
\hline miR-139-5p & $\downarrow$ & $\mathrm{n} / \mathrm{d}$ & $\mathrm{n} / \mathrm{d}$ & [54] & $\operatorname{miR}-210$ & $\uparrow$ & $\mathrm{n} / \mathrm{d}$ & $\mathrm{n} / \mathrm{d}$ & [100] \\
\hline $\operatorname{miR}-142$ & $\downarrow$ & $\mathrm{n} / \mathrm{d}$ & $\mathrm{n} / \mathrm{d}$ & [69] & $\operatorname{miR}-218^{*}$ & & $\mathrm{n} / \mathrm{d}$ & $\mathrm{n} / \mathrm{d}$ & [30] \\
\hline miR-143 & $\downarrow$ & $\downarrow$ & $\downarrow$ & [22] & $\operatorname{miR}-222-3 p$ & $\uparrow$ & $\uparrow$ & $\mathrm{n} / \mathrm{d}$ & {$[47,52]$} \\
\hline $\operatorname{miR}-144-3 p$ & $\downarrow$ & $\downarrow$ & $\mathrm{n} / \mathrm{d}$ & [85] & miR-301 & $\uparrow$ & $\mathrm{n} / \mathrm{d}$ & $\mathrm{n} / \mathrm{d}$ & [57] \\
\hline miR-152 & $\downarrow$ & $\mathrm{n} / \mathrm{d}$ & $\mathrm{n} / \mathrm{d}$ & [38] & miR-373 & $\uparrow$ & $\uparrow$ & $\mathrm{n} / \mathrm{d}$ & {$[49,99]$} \\
\hline miR-183-5p & $\downarrow$ & $\mathrm{n} / \mathrm{d}$ & $\mathrm{n} / \mathrm{d}$ & [98] & miR-429 & $\uparrow$ & $\mathrm{n} / \mathrm{d}$ & $\mathrm{n} / \mathrm{d}$ & {$[77,102]$} \\
\hline miR-184 & $\downarrow$ & $\mathrm{n} / \mathrm{d}$ & $\mathrm{n} / \mathrm{d}$ & [28] & $\mathrm{miR}-449$ & $\uparrow$ & $\mathrm{n} / \mathrm{d}$ & $\mathrm{n} / \mathrm{d}$ & [30] \\
\hline miR-195 & $\downarrow$ & $\downarrow$ & $\mathrm{n} / \mathrm{d}$ & {$[78,108]$} & miR-449a & $\mathrm{n} / \mathrm{d}$ & $\mathrm{n} / \mathrm{d}$ & $\uparrow$ & [77] \\
\hline miR-196a & $\downarrow$ & $\mathrm{n} / \mathrm{d}$ & $\mathrm{n} / \mathrm{d}$ & [23] & miR-486-5p & $\uparrow$ & $\mathrm{n} / \mathrm{d}$ & $\uparrow$ & [112] \\
\hline
\end{tabular}


Table 2. Cont.

\begin{tabular}{|c|c|c|c|c|c|c|c|c|c|}
\hline MiRNA & $\underset{\text { Tissue }}{\text { Human }}$ & $\begin{array}{l}\text { Cell } \\
\text { Line }\end{array}$ & $\begin{array}{c}\text { Circulating } \\
\text { miRNA }\end{array}$ & Ref. & MiRNA & $\begin{array}{l}\text { Human } \\
\text { Tissue }\end{array}$ & $\begin{array}{l}\text { Cell } \\
\text { Line }\end{array}$ & $\begin{array}{c}\text { Circulating } \\
\text { miRNA }\end{array}$ & Ref. \\
\hline miR-199a-3p & $\downarrow$ & $\mathrm{n} / \mathrm{d}$ & $\mathrm{n} / \mathrm{d}$ & [90] & miR-494-3p & $\uparrow$ & $\mathrm{n} / \mathrm{d}$ & $\mathrm{n} / \mathrm{d}$ & [117] \\
\hline miR-199a/b-5p & $\downarrow$ & $\downarrow$ & $\mathrm{n} / \mathrm{d}$ & [93] & miR-499 & $\uparrow$ & $\mathrm{n} / \mathrm{d}$ & $\mathrm{n} / \mathrm{d}$ & [78] \\
\hline miR-202 & $\downarrow$ & $\downarrow$ & $\mathrm{n} / \mathrm{d}$ & {$[25,94]$} & miR-522 & 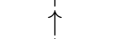 & & $\mathrm{n} / \mathrm{d}$ & [132] \\
\hline miR-204 & $\downarrow$ & $\mathrm{n} / \mathrm{d}$ & $\mathrm{n} / \mathrm{d}$ & {$[18,30]$} & miR-544a & $\uparrow$ & $\uparrow$ & $\mathrm{n} / \mathrm{d}$ & [111] \\
\hline miR-205* & $\mathrm{n} / \mathrm{d}$ & $\mathrm{n} / \mathrm{d}$ & $\downarrow$ & {$[110,118]$} & miR-652 & $\uparrow$ & $\mathrm{n} / \mathrm{d}$ & $\mathrm{n} / \mathrm{d}$ & [72] \\
\hline miR-206 & & $\downarrow$ & $\mathrm{n} / \mathrm{d}$ & [124] & miR-940 & $\uparrow$ & & $\mathrm{n} / \mathrm{d}$ & [115] \\
\hline $\operatorname{miR}-214-3 p$ & $\downarrow$ & $\downarrow$ & $\mathrm{n} / \mathrm{d}$ & {$[36,82]$} & miR-1202 & $\uparrow$ & $\mathrm{n} / \mathrm{d}$ & $\mathrm{n} / \mathrm{d}$ & [23] \\
\hline $\operatorname{miR}-218^{*}$ & $\downarrow$ & $\downarrow$ & $\mathrm{n} / \mathrm{d}$ & [125] & $\mathrm{miR}-1228$ & $\mathrm{n} / \mathrm{d}$ & $\mathrm{n} / \mathrm{d}$ & $\uparrow$ & [77] \\
\hline miR-219-5p & $\downarrow$ & $\mathrm{n} / \mathrm{d}$ & $\mathrm{n} / \mathrm{d}$ & [87] & & & & & \\
\hline miR-301b & $\mathrm{n} / \mathrm{d}$ & $\mathrm{n} / \mathrm{d}$ & $\downarrow$ & {$[77]$} & & & & & \\
\hline miR-302a-5p & $\downarrow$ & $\mathrm{n} / \mathrm{d}$ & $\mathrm{n} / \mathrm{d}$ & [126] & & & & & \\
\hline miR-302c-3p & 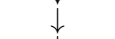 & $\mathrm{n} / \mathrm{d}$ & $\mathrm{n} / \mathrm{d}$ & [67] & & & & & \\
\hline miR-320a & $\downarrow$ & $\mathrm{n} / \mathrm{d}$ & $\mathrm{n} / \mathrm{d}$ & [103] & & & & & \\
\hline miR-326 & $\downarrow$ & $\downarrow$ & $\mathrm{n} / \mathrm{d}$ & [55] & & & & & \\
\hline miR-340-5p & 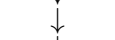 & $\mathrm{n} / \mathrm{d}$ & $\mathrm{n} / \mathrm{d}$ & [103] & & & & & \\
\hline miR-361 & $\downarrow$ & $\downarrow$ & $\mathrm{n} / \mathrm{d}$ & {$[41]$} & & & & & \\
\hline miR-363 & $\downarrow$ & $\downarrow$ & $\mathrm{n} / \mathrm{d}$ & [83] & & & & & \\
\hline miR-365 & & $\downarrow$ & $\mathrm{n} / \mathrm{d}$ & [80] & & & & & \\
\hline miR-367-3p & & $\mathrm{n} / \mathrm{d}$ & $\mathrm{n} / \mathrm{d}$ & [126] & & & & & \\
\hline miR-372 & 1 & $\mathrm{n} / \mathrm{d}$ & $\mathrm{n} / \mathrm{d}$ & [130] & & & & & \\
\hline miR-381 & & $\downarrow$ & $\mathrm{n} / \mathrm{d}$ & [79] & & & & & \\
\hline $\mathrm{miR}-424$ & & $\downarrow$ & $\mathrm{n} / \mathrm{d}$ & {$[34,58,66]$} & & & & & \\
\hline miR-449a & & $\mathrm{n} / \mathrm{d}$ & $\mathrm{n} / \mathrm{d}$ & [127] & & & & & \\
\hline miR-490-3p & & $\mathrm{n} / \mathrm{d}$ & $\mathrm{n} / \mathrm{d}$ & {$[64,70]$} & & & & & \\
\hline miR-499a & $\downarrow$ & $\downarrow$ & $\mathrm{n} / \mathrm{d}$ & [45] & & & & & \\
\hline miR-505 & $j$ & $\mathrm{n} / \mathrm{d}$ & $\mathrm{n} / \mathrm{d}$ & [131] & & & & & \\
\hline miR-513 & $\downarrow$ & $\mathrm{n} / \mathrm{d}$ & $\mathrm{n} / \mathrm{d}$ & [34] & & & & & \\
\hline miR-543 & $\downarrow$ & $\downarrow$ & $\mathrm{n} / \mathrm{d}$ & [20] & & & & & \\
\hline miR-548c & $\downarrow$ & $\mathrm{n} / \mathrm{d}$ & $\mathrm{n} / \mathrm{d}$ & [71] & & & & & \\
\hline miR-589-5p & $\downarrow$ & $\mathrm{n} / \mathrm{d}$ & $\mathrm{n} / \mathrm{d}$ & [86] & & & & & \\
\hline miR-646 & $\downarrow$ & $\downarrow$ & $\mathrm{n} / \mathrm{d}$ & [113] & & & & & \\
\hline miR-873 & $\downarrow$ & $\downarrow$ & $\mathrm{n} / \mathrm{d}$ & [84] & & & & & \\
\hline miR-1271-5p & $\downarrow$ & $\downarrow$ & $\mathrm{n} / \mathrm{d}$ & {$[74,88]$} & & & & & \\
\hline
\end{tabular}

$\downarrow$-downregulated in endometrial cancer compared to normal cells, $\uparrow$ - upregulated in endometrial cancer compared to normal cells, $\mathrm{n} / \mathrm{d}$ - no data, * - inconsistent data of miRNAs expression.

\subsection{MiRNAs Regulating Endometrial Cancer (EC) Invasiveness and Metastasis}

Further, we collected data from articles investigating the role of miRNAs in the regulation of EC invasiveness and metastasis. We identified 132 articles demonstrating the role of 97 miRNAs in the regulation of migration, invasiveness, and EMT of endometrial cancer cell lines in vitro [18-28,30-34,36,39-46,49-56,58,62-74,79,80,82,83,8588,91-98,100-103,105-109,111-117,119,121-131,133-173]. From them, 69 miRNAs act as tumor-suppressor miRNAs and 26 are oncomiRNAs, and two miRNAs (miR-130b and miR-200c) have an unclear role due to inconsistent data (Figure 3).

Included studies involved established EC cell lines, including Ishikawa, HEC-1A, HEC-1B, HHUA, AN3CA, ECC-1, RL-95-2, KLE, SPAC-1-L, HEC-50, HOUA-I, and JEC cell line (EC cell lines have been reviewed by Van Nyen et al. [174]). Transient upregulation of a given miRNA with synthetic miRNA or downregulation with complementary antimiRNA revealed regulation of tumor cell migration and invasiveness in vitro assays by 97 miRNAs. Further, luciferase reporter assays confirmed direct binding of 102 targets by miRNAs regulating invasiveness of EC cells creating a complex regulatory network. Tumor-suppressor miRNAs that are downregulated in EC were identified to bind 77 targets, including some of the well-known oncogenes. OncomiRNAs were identified to promote EC cell migration and invasiveness by binding 25 targets.

Moreover, 35 studies investigated the role of miRNAs in the regulation of tumor growth and EC metastasis in vivo in murine models $[18,19,24,36,41,42,44,45,49,51-53,56,62$, $69,70,72,73,92,93,101,111,117,119,122,126,128,136,137,144,145,151,157,160,166]$. Eight miRNAs were identified as oncomiRNAs in vivo (miR-106a, miR-107-5p, miR-130b, miR-183, miR-222-3p, miR-494-3p, miR-544a, and miR-652) and 27 as tumor suppressor miRNAs (miR-23a, miR-23b, miR-26a, miR-29a-5p, miR-29b, miR-34a, miR-34b, miR-99a, miR-101, miR-129, miR-142, miR-148b, miR-194, miR-199a/b-5p, miR-200c, miR-204, miR-214-3p, miR-302a-5p, miR-326, miR-361, miR-367-3p, miR-372, miR-449a, miR-490-3p, miR-499a, 
miR-505, and miR-1827). All but miR-148b and miR-652 were found to regulate primary tumor growth. Moreover, miR-29b, miR-148b, miR-194, miR-199a/b-5p and miR-214-3p were identified to suppress tumor metastasis while miR-652 were found to promote this process. Most of the studies used xenograft assays with human HEC-1B or Ishikawa EC cell lines in immunocompromised mice (Table 3).

\section{The regulation of invasiveness by miRNAs}

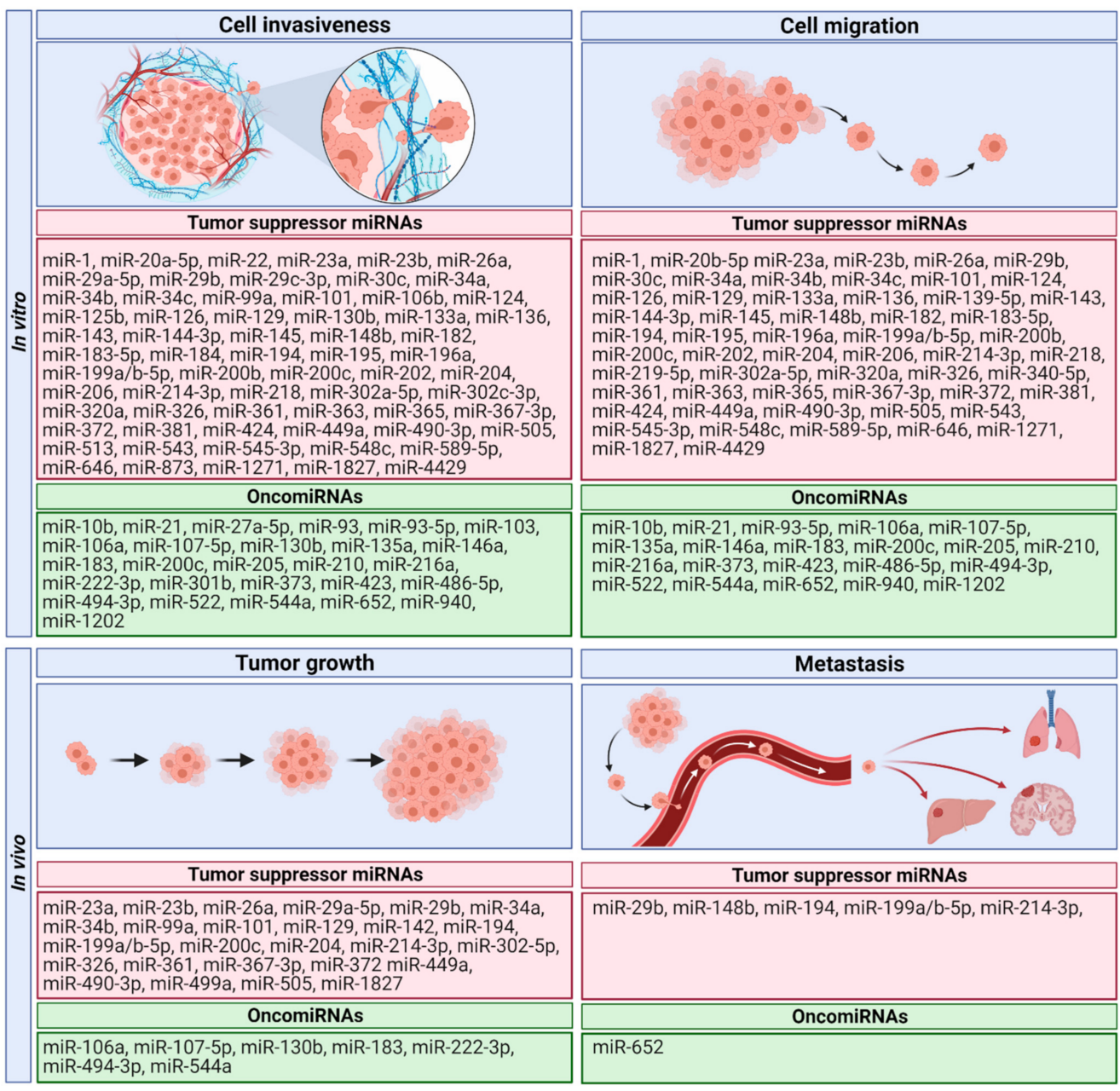

Figure 3. miRNAs regulating cell invasiveness and cell migration in vitro as well as tumor growth, and metastasis in vivo. Figure was created using Biorender.com.

Table 3. The role of miRNAs in invasion and metastasis in vitro and in vivo.

\begin{tabular}{|c|c|c|c|c|c|c|c|}
\hline MiRNA & Target ${ }^{1}$ & Migration & Invasion & EMT & $\begin{array}{l}\text { Tumor Growth } \\
\text { In Vivo }\end{array}$ & $\begin{array}{l}\text { Metastasis } \\
\text { In Vivo }\end{array}$ & Ref. \\
\hline miR-1 & PDE7A & $\downarrow$ & $\downarrow$ & $\mathrm{n} / \mathrm{d}$ & $\mathrm{n} / \mathrm{d}$ & $\mathrm{n} / \mathrm{d}$ & [97] \\
\hline miR-20a-5p & STAT3 & $\mathrm{n} / \mathrm{d}$ & $\downarrow$ & $\downarrow$ & $\mathrm{n} / \mathrm{d}$ & $\mathrm{n} / \mathrm{d}$ & {$[40]$} \\
\hline $\mathrm{miR}-20 \mathrm{~b}-5 \mathrm{p}$ & H19 & $\downarrow$ & $\mathrm{n} / \mathrm{d}$ & $\mathrm{n} / \mathrm{d}$ & $\mathrm{n} / \mathrm{d}$ & $\mathrm{n} / \mathrm{d}$ & [116] \\
\hline miR-22 & $\mathrm{ER} \alpha$ & $\mathrm{n} / \mathrm{d}$ & $\downarrow$ & $\mathrm{n} / \mathrm{d}$ & $\mathrm{n} / \mathrm{d}$ & $\mathrm{n} / \mathrm{d}$ & {$[121,156]$} \\
\hline miR-23a & SIX1 & $\downarrow$ & $\downarrow$ & $\mathrm{n} / \mathrm{d}$ & $\downarrow$ & $\mathrm{n} / \mathrm{d}$ & [128] \\
\hline $\operatorname{miR}-23 b$ & MACC1 & $\downarrow$ & $\downarrow$ & $\mathrm{n} / \mathrm{d}$ & $\downarrow$ & $\mathrm{n} / \mathrm{d}$ & [137] \\
\hline miR-26a & EZH2 & $\downarrow$ & $\downarrow$ & $\downarrow$ & $\downarrow$ & $\mathrm{n} / \mathrm{d}$ & [42] \\
\hline $\operatorname{miR}-29 a-5 p$ & TPX2 & $\mathrm{n} / \mathrm{d}$ & $\downarrow$ & $\mathrm{n} / \mathrm{d}$ & $\downarrow$ & $\mathrm{n} / \mathrm{d}$ & [44] \\
\hline miR-29b & VEGFA, PTEN & $\downarrow$ & $\downarrow$ & $\mathrm{n} / \mathrm{d}$ & $\downarrow$ & $\downarrow$ & {$[24,150]$} \\
\hline
\end{tabular}


Table 3. Cont.

\begin{tabular}{|c|c|c|c|c|c|c|c|}
\hline MiRNA & Target ${ }^{1}$ & Migration & Invasion & EMT & $\begin{array}{l}\text { Tumor Growth } \\
\text { In Vivo }\end{array}$ & $\begin{array}{l}\text { Metastasis } \\
\text { In Vivo }\end{array}$ & Ref. \\
\hline $\operatorname{miR}-29 c-3 p$ & KDM5B & $\mathrm{n} / \mathrm{d}$ & $\downarrow$ & $\mathrm{n} / \mathrm{d}$ & $\mathrm{n} / \mathrm{d}$ & $\mathrm{n} / \mathrm{d}$ & [154] \\
\hline miR-30c & MTA-1 & $\downarrow$ & $\downarrow$ & $\mathrm{n} / \mathrm{d}$ & $\mathrm{n} / \mathrm{d}$ & $\mathrm{n} / \mathrm{d}$ & {$[96,173]$} \\
\hline $\operatorname{miR}-34 a$ & $\begin{array}{l}\text { Notch1, L1CAM, } \\
\text { MMSET }\end{array}$ & $\downarrow$ & $\downarrow$ & $\downarrow$ & $\downarrow$ & $\mathrm{n} / \mathrm{d}$ & {$[34,166,170]$} \\
\hline $\operatorname{miR}-34 b$ & $\mathrm{n} / \mathrm{d}$ & $\downarrow$ & $\downarrow$ & $\mathrm{n} / \mathrm{d}$ & $\downarrow$ & $\mathrm{n} / \mathrm{d}$ & {$[39,101]$} \\
\hline $\mathrm{miR}-34 \mathrm{c}$ & IL-6R & $\downarrow$ & $\downarrow$ & $\mathrm{n} / \mathrm{d}$ & $\mathrm{n} / \mathrm{d}$ & $\mathrm{n} / \mathrm{d}$ & {$[43,123]$} \\
\hline $\operatorname{miR}-99 a$ & AKT1, mTOR & $\mathrm{n} / \mathrm{d}$ & $\downarrow$ & $\mathrm{n} / \mathrm{d}$ & $\downarrow$ & $\mathrm{n} / \mathrm{d}$ & [51] \\
\hline miR-101 & $\begin{array}{l}\text { COX-2, EZH2, } \\
\text { MCL-1, FOS, } \\
\text { mTOR }\end{array}$ & $\downarrow$ & $\downarrow$ & $\downarrow$ & $\downarrow$ & $\mathrm{n} / \mathrm{d}$ & $\begin{array}{l}{[46,56,106,} \\
119]\end{array}$ \\
\hline miR-106b & TWIST1 & $\mathrm{n} / \mathrm{d}$ & $\downarrow$ & $\downarrow$ & $\mathrm{n} / \mathrm{d}$ & $\mathrm{n} / \mathrm{d}$ & [140] \\
\hline $\operatorname{miR}-124$ & $\begin{array}{c}\text { STAT3, IQGAP1, } \\
\text { ITGB3 }\end{array}$ & $\downarrow$ & $\downarrow$ & $\downarrow$ & $\mathrm{n} / \mathrm{d}$ & $\mathrm{n} / \mathrm{d}$ & {$[33,50,167]$} \\
\hline $\operatorname{miR}-125 b$ & ERBB2 & $\mathrm{n} / \mathrm{d}$ & $\downarrow$ & $\mathrm{n} / \mathrm{d}$ & $\mathrm{n} / \mathrm{d}$ & $\mathrm{n} / \mathrm{d}$ & {$[63,161]$} \\
\hline miR-126 & IRS1 & $\downarrow$ & $\downarrow$ & $\mathrm{n} / \mathrm{d}$ & $\mathrm{n} / \mathrm{d}$ & $\mathrm{n} / \mathrm{d}$ & [109] \\
\hline miR-129 & GSK-3 $\beta$ & $\downarrow$ & $\downarrow$ & $\mathrm{n} / \mathrm{d}$ & $\downarrow$ & $\mathrm{n} / \mathrm{d}$ & [136] \\
\hline miR-130b* & ZEB1 & $\mathrm{n} / \mathrm{d}$ & $\downarrow$ & $\downarrow$ & $\mathrm{n} / \mathrm{d}$ & $\mathrm{n} / \mathrm{d}$ & [32] \\
\hline miR-133a & PDE7A & $\downarrow$ & $\downarrow$ & $\mathrm{n} / \mathrm{d}$ & $\mathrm{n} / \mathrm{d}$ & $\mathrm{n} / \mathrm{d}$ & [97] \\
\hline miR-136 & HMGA2 & $\downarrow$ & $\downarrow$ & $\mathrm{n} / \mathrm{d}$ & $\mathrm{n} / \mathrm{d}$ & $\mathrm{n} / \mathrm{d}$ & [65] \\
\hline miR-139-5p & HOXA10 & $\downarrow$ & $\mathrm{n} / \mathrm{d}$ & $\mathrm{n} / \mathrm{d}$ & $\mathrm{n} / \mathrm{d}$ & $\mathrm{n} / \mathrm{d}$ & [54] \\
\hline miR-142 & CCND1 & $\mathrm{n} / \mathrm{d}$ & $\mathrm{n} / \mathrm{d}$ & $\mathrm{n} / \mathrm{d}$ & $\downarrow$ & $\mathrm{n} / \mathrm{d}$ & [69] \\
\hline miR-143 & MAPK1 & $\downarrow$ & $\downarrow$ & $\mathrm{n} / \mathrm{d}$ & $\mathrm{n} / \mathrm{d}$ & $\mathrm{n} / \mathrm{d}$ & [22] \\
\hline miR-144-3p & EZH2 & $\downarrow$ & $\downarrow$ & $\mathrm{n} / \mathrm{d}$ & $\mathrm{n} / \mathrm{d}$ & $\mathrm{n} / \mathrm{d}$ & [85] \\
\hline miR-145 & SOX11 & $\downarrow$ & $\downarrow$ & $\mathrm{n} / \mathrm{d}$ & $\mathrm{n} / \mathrm{d}$ & $\mathrm{n} / \mathrm{d}$ & [21] \\
\hline miR-148b & DNMT1 & $\downarrow$ & $\downarrow$ & $\mathrm{n} / \mathrm{d}$ & $\mathrm{n} / \mathrm{d}$ & $\downarrow$ & [151] \\
\hline miR-182 & FOXO1 & $\downarrow$ & $\downarrow$ & $\mathrm{n} / \mathrm{d}$ & $\mathrm{n} / \mathrm{d}$ & $\mathrm{n} / \mathrm{d}$ & {$[142,163]$} \\
\hline miR-183-5p & Ezrin & $\downarrow$ & $\downarrow$ & $\downarrow$ & $\mathrm{n} / \mathrm{d}$ & $\mathrm{n} / \mathrm{d}$ & [98] \\
\hline miR-184 & CDC25A & $\mathrm{n} / \mathrm{d}$ & $\downarrow$ & $\mathrm{n} / \mathrm{d}$ & $\mathrm{n} / \mathrm{d}$ & $\mathrm{n} / \mathrm{d}$ & [28] \\
\hline miR-194 & BMI-1, Sox3 & $\downarrow$ & $\downarrow$ & $\downarrow$ & $\downarrow$ & $\downarrow$ & {$[141,145]$} \\
\hline miR-195 & SOX4, GPER & $\downarrow$ & $\downarrow$ & $\downarrow$ & $\mathrm{n} / \mathrm{d}$ & $\mathrm{n} / \mathrm{d}$ & {$[108,139]$} \\
\hline miR-196a & $\mathrm{n} / \mathrm{d}$ & $\downarrow$ & $\downarrow$ & $\mathrm{n} / \mathrm{d}$ & $\mathrm{n} / \mathrm{d}$ & $\mathrm{n} / \mathrm{d}$ & [23] \\
\hline miR-199a/b-5p & FAM83B & $\downarrow$ & $\downarrow$ & $\downarrow$ & $\downarrow$ & $\downarrow$ & [93] \\
\hline $\mathrm{miR}-200 \mathrm{~b}$ & $\begin{array}{l}\text { TIMP2, PTEN, } \\
\text { ZEB2 }\end{array}$ & $\downarrow$ & $\downarrow$ & $\mathrm{n} / \mathrm{d}$ & $\mathrm{n} / \mathrm{d}$ & $\mathrm{n} / \mathrm{d}$ & {$[31,102,133]$} \\
\hline $\operatorname{miR}-200 c^{*}$ & $\begin{array}{c}\text { TUBB3, BMI-1, } \\
\text { MSN, FN1, TrkB, } \\
\text { ARHGAP19, } \\
\text { LEPR }\end{array}$ & $\downarrow$ & $\downarrow$ & $\downarrow$ & $\downarrow$ & $\mathrm{n} / \mathrm{d}$ & {$[138,146,152]$} \\
\hline miR-202 & FGF2, TIMD4 & $\downarrow$ & $\downarrow$ & $\downarrow$ & $\mathrm{n} / \mathrm{d}$ & $\mathrm{n} / \mathrm{d}$ & {$[25,94]$} \\
\hline miR-204 & TrkB, FOXC1 & $\downarrow$ & $\downarrow$ & $\mathrm{n} / \mathrm{d}$ & $\downarrow$ & $\mathrm{n} / \mathrm{d}$ & {$[18,30]$} \\
\hline miR-206 & HDAC6, ER $\alpha$ & $\downarrow$ & $\downarrow$ & $\mathrm{n} / \mathrm{d}$ & $\mathrm{n} / \mathrm{d}$ & $\mathrm{n} / \mathrm{d}$ & {$[124,168]$} \\
\hline miR-214-3p & $\begin{array}{l}\text { TWIST1, } \\
\text { HMGA1 }\end{array}$ & $\downarrow$ & $\downarrow$ & $\downarrow$ & $\downarrow$ & $\downarrow$ & {$[36,82]$} \\
\hline miR-218 & ADD2 & $\downarrow$ & $\downarrow$ & $\mathrm{n} / \mathrm{d}$ & $\mathrm{n} / \mathrm{d}$ & $\mathrm{n} / \mathrm{d}$ & [125] \\
\hline miR-219-5p & COX-2 & $\downarrow$ & $\mathrm{n} / \mathrm{d}$ & $\mathrm{n} / \mathrm{d}$ & $\mathrm{n} / \mathrm{d}$ & $\mathrm{n} / \mathrm{d}$ & [87] \\
\hline miR-302a-5p & HMGA2 & $\downarrow$ & $\downarrow$ & $\mathrm{n} / \mathrm{d}$ & $\downarrow$ & $\mathrm{n} / \mathrm{d}$ & [126] \\
\hline $\operatorname{miR}-302 c-3 p$ & ZFX, YKL-40 & $\mathrm{n} / \mathrm{d}$ & $\downarrow$ & $\mathrm{n} / \mathrm{d}$ & $\mathrm{n} / \mathrm{d}$ & $\mathrm{n} / \mathrm{d}$ & [67] \\
\hline miR-320a & eIF4E & $\downarrow$ & $\downarrow$ & $\downarrow$ & $\mathrm{n} / \mathrm{d}$ & $\mathrm{n} / \mathrm{d}$ & [103] \\
\hline miR-326 & TWIST1, GPR91 & $\downarrow$ & $\downarrow$ & $\downarrow$ & $\downarrow$ & $\mathrm{n} / \mathrm{d}$ & {$[55,144]$} \\
\hline miR-340-5p & eIF4E & $\downarrow$ & $\mathrm{n} / \mathrm{d}$ & $\downarrow$ & $\mathrm{n} / \mathrm{d}$ & $\mathrm{n} / \mathrm{d}$ & [103] \\
\hline miR-361 & TWIST, STAT3 & $\downarrow$ & $\downarrow$ & $\mathrm{n} / \mathrm{d}$ & $\downarrow$ & $\mathrm{n} / \mathrm{d}$ & {$[41,169]$} \\
\hline miR-363 & SOX4 & $\downarrow$ & $\downarrow$ & $\mathrm{n} / \mathrm{d}$ & $\mathrm{n} / \mathrm{d}$ & $\mathrm{n} / \mathrm{d}$ & [83] \\
\hline miR-365 & EZH2, FOS & $\downarrow$ & $\downarrow$ & $\downarrow$ & $\mathrm{n} / \mathrm{d}$ & $\mathrm{n} / \mathrm{d}$ & {$[80]$} \\
\hline $\operatorname{miR}-367-3 p$ & HMGA2 & $\downarrow$ & $\downarrow$ & $\mathrm{n} / \mathrm{d}$ & $\downarrow$ & $\mathrm{n} / \mathrm{d}$ & [126] \\
\hline miR-372 & RhoC & $\downarrow$ & $\downarrow$ & $\mathrm{n} / \mathrm{d}$ & $\downarrow$ & $\mathrm{n} / \mathrm{d}$ & [130] \\
\hline
\end{tabular}


Table 3. Cont.

\begin{tabular}{|c|c|c|c|c|c|c|c|}
\hline MiRNA & Target ${ }^{1}$ & Migration & Invasion & EMT & $\begin{array}{l}\text { Tumor Growth } \\
\text { In Vivo }\end{array}$ & $\begin{array}{l}\text { Metastasis } \\
\text { In Vivo }\end{array}$ & Ref. \\
\hline miR-381 & IGF-1R, E2F3 & $\downarrow$ & $\downarrow$ & $\mathrm{n} / \mathrm{d}$ & $\mathrm{n} / \mathrm{d}$ & $\mathrm{n} / \mathrm{d}$ & {$[79,148]$} \\
\hline $\operatorname{miR}-424$ & $\begin{array}{l}\text { IGF-1R, E2F6, } \\
\text { MMSET }\end{array}$ & $\downarrow$ & $\downarrow$ & $\downarrow$ & $\mathrm{n} / \mathrm{d}$ & $\mathrm{n} / \mathrm{d}$ & {$[34,58,66]$} \\
\hline miR-449a & $\begin{array}{l}\text { NDRG1, SRC, } \\
\text { MET }\end{array}$ & $\downarrow$ & $\downarrow$ & $\mathrm{n} / \mathrm{d}$ & $\downarrow$ & $\mathrm{n} / \mathrm{d}$ & $\begin{array}{c}{[127,143,147,} \\
160,172]\end{array}$ \\
\hline miR-490-3p & $\mathrm{TGF} \alpha, \mathrm{SP} 1$ & $\downarrow$ & $\downarrow$ & $\mathrm{n} / \mathrm{d}$ & $\downarrow$ & $\mathrm{n} / \mathrm{d}$ & {$[64,70]$} \\
\hline $\operatorname{miR}-499 a$ & VAV3 & $\mathrm{n} / \mathrm{d}$ & $\mathrm{n} / \mathrm{d}$ & $\mathrm{n} / \mathrm{d}$ & $\downarrow$ & $\mathrm{n} / \mathrm{d}$ & {$[45]$} \\
\hline miR-505 & $\mathrm{TGF} \alpha$ & $\downarrow$ & $\downarrow$ & $\mathrm{n} / \mathrm{d}$ & $\downarrow$ & $\mathrm{n} / \mathrm{d}$ & [131] \\
\hline miR-513 & MMSET & $\mathrm{n} / \mathrm{d}$ & $\downarrow$ & $\downarrow$ & $\mathrm{n} / \mathrm{d}$ & $\mathrm{n} / \mathrm{d}$ & [34] \\
\hline miR-543 & FAK, TWIST1 & $\downarrow$ & $\downarrow$ & $\mathrm{n} / \mathrm{d}$ & $\mathrm{n} / \mathrm{d}$ & $\mathrm{n} / \mathrm{d}$ & [20] \\
\hline miR-545-3p & VEGF & $\downarrow$ & $\downarrow$ & $\mathrm{n} / \mathrm{d}$ & $\mathrm{n} / \mathrm{d}$ & $\mathrm{n} / \mathrm{d}$ & [165] \\
\hline miR-548c & TWIST & $\downarrow$ & $\downarrow$ & $\mathrm{n} / \mathrm{d}$ & $\mathrm{n} / \mathrm{d}$ & $\mathrm{n} / \mathrm{d}$ & [71] \\
\hline miR-589-5p & TRIP6 & $\downarrow$ & $\downarrow$ & $\mathrm{n} / \mathrm{d}$ & $\mathrm{n} / \mathrm{d}$ & $\mathrm{n} / \mathrm{d}$ & [86] \\
\hline $\mathrm{miR}-646$ & NPM1 & $\downarrow$ & $\downarrow$ & $\mathrm{n} / \mathrm{d}$ & $\mathrm{n} / \mathrm{d}$ & $\mathrm{n} / \mathrm{d}$ & {$[113,155]$} \\
\hline miR-873 & HDGF & $\mathrm{n} / \mathrm{d}$ & $\downarrow$ & $\mathrm{n} / \mathrm{d}$ & $\mathrm{n} / \mathrm{d}$ & $\mathrm{n} / \mathrm{d}$ & [84] \\
\hline miR-1271 & $\begin{array}{l}\text { LDHA, } \\
\text { CTNND1 }\end{array}$ & $\downarrow$ & $\downarrow$ & $\mathrm{n} / \mathrm{d}$ & $\mathrm{n} / \mathrm{d}$ & $\mathrm{n} / \mathrm{d}$ & {$[74,88]$} \\
\hline miR-1827 & ТРPР3 & $\downarrow$ & $\downarrow$ & $\mathrm{n} / \mathrm{d}$ & $\downarrow$ & $\mathrm{n} / \mathrm{d}$ & [157] \\
\hline miR-4429 & SNHG12 & $\downarrow$ & $\downarrow$ & $\mathrm{n} / \mathrm{d}$ & $\mathrm{n} / \mathrm{d}$ & $\mathrm{n} / \mathrm{d}$ & [134] \\
\hline miR-10b & HOXB3 & $\uparrow$ & $\uparrow$ & $\mathrm{n} / \mathrm{d}$ & $\mathrm{n} / \mathrm{d}$ & $\mathrm{n} / \mathrm{d}$ & [129] \\
\hline miR-21 & $\mathrm{n} / \mathrm{d}$ & $\uparrow$ & $\uparrow$ & $\mathrm{n} / \mathrm{d}$ & $\mathrm{n} / \mathrm{d}$ & $\mathrm{n} / \mathrm{d}$ & [107] \\
\hline miR-27a-5p & SMAD4 & $\mathrm{n} / \mathrm{d}$ & $\uparrow$ & $\mathrm{n} / \mathrm{d}$ & $\mathrm{n} / \mathrm{d}$ & $\mathrm{n} / \mathrm{d}$ & [135] \\
\hline miR-93 & FOXA1 & $\mathrm{n} / \mathrm{d}$ & $\uparrow$ & $\uparrow$ & $\mathrm{n} / \mathrm{d}$ & $\mathrm{n} / \mathrm{d}$ & [27] \\
\hline miR-93-5p & ZBTB7A & $\uparrow$ & $\uparrow$ & $\mathrm{n} / \mathrm{d}$ & $\mathrm{n} / \mathrm{d}$ & $\mathrm{n} / \mathrm{d}$ & {$[95,105]$} \\
\hline miR-103 & TIMP3 & $\mathrm{n} / \mathrm{d}$ & $\uparrow$ & $\mathrm{n} / \mathrm{d}$ & $\mathrm{n} / \mathrm{d}$ & $\mathrm{n} / \mathrm{d}$ & [164] \\
\hline miR-106a & BCL2L11 & $\uparrow$ & $\uparrow$ & $\mathrm{n} / \mathrm{d}$ & $\uparrow$ & $\mathrm{n} / \mathrm{d}$ & [73] \\
\hline miR-107-5p & $\mathrm{ER} \alpha$ & $\uparrow$ & $\uparrow$ & $\mathrm{n} / \mathrm{d}$ & $\uparrow$ & $\mathrm{n} / \mathrm{d}$ & [19] \\
\hline $\operatorname{miR}-130 b$ * & $\begin{array}{c}\text { DICER1, } \\
\text { BHLHE40/41 }\end{array}$ & $\mathrm{n} / \mathrm{d}$ & $\uparrow$ & $\uparrow$ & $\uparrow$ & $\mathrm{n} / \mathrm{d}$ & {$[122,171]$} \\
\hline miR-135a & PTEN & $\uparrow$ & $\uparrow$ & $\mathrm{n} / \mathrm{d}$ & $\mathrm{n} / \mathrm{d}$ & $\mathrm{n} / \mathrm{d}$ & [159] \\
\hline miR-146a & $\mathrm{n} / \mathrm{d}$ & $\uparrow$ & $\uparrow$ & $\mathrm{n} / \mathrm{d}$ & $\mathrm{n} / \mathrm{d}$ & $\mathrm{n} / \mathrm{d}$ & [114] \\
\hline miR-183 & $\begin{array}{c}\text { CPEB1, MMP-9, } \\
\text { FOXO1 }\end{array}$ & $\uparrow$ & $\uparrow$ & $\uparrow$ & $\uparrow$ & $\mathrm{n} / \mathrm{d}$ & {$[62,92,142]$} \\
\hline miR-200a & FOXA2, PTEN & $\mathrm{n} / \mathrm{d}$ & $\mathrm{n} / \mathrm{d}$ & $\uparrow$ & $\mathrm{n} / \mathrm{d}$ & $\mathrm{n} / \mathrm{d}$ & {$[102,158]$} \\
\hline $\operatorname{miR}-200 c^{*}$ & $\begin{array}{l}\text { PTENP1, PTEN, } \\
\text { MALAT1 }\end{array}$ & $\uparrow$ & $\uparrow$ & $\uparrow$ & $\mathrm{n} / \mathrm{d}$ & $\mathrm{n} / \mathrm{d}$ & {$[26,53]$} \\
\hline miR-205 & ESRRG, PTEN & $\uparrow$ & $\uparrow$ & $\uparrow$ & $\mathrm{n} / \mathrm{d}$ & $\mathrm{n} / \mathrm{d}$ & {$[68,91,149]$} \\
\hline miR-210 & NFIX & $\uparrow$ & $\uparrow$ & $\mathrm{n} / \mathrm{d}$ & $\mathrm{n} / \mathrm{d}$ & $\mathrm{n} / \mathrm{d}$ & [100] \\
\hline miR-216a & PD-L1 & $\uparrow$ & $\uparrow$ & $\mathrm{n} / \mathrm{d}$ & $\mathrm{n} / \mathrm{d}$ & $\mathrm{n} / \mathrm{d}$ & [162] \\
\hline miR-222-3p & ERa & $\mathrm{n} / \mathrm{d}$ & $\uparrow$ & $\mathrm{n} / \mathrm{d}$ & $\uparrow$ & $\mathrm{n} / \mathrm{d}$ & [52] \\
\hline miR-301b & BHLHE40/41 & $\mathrm{n} / \mathrm{d}$ & $\uparrow$ & $\uparrow$ & $\mathrm{n} / \mathrm{d}$ & $\mathrm{n} / \mathrm{d}$ & [171] \\
\hline $\operatorname{miR}-373$ & LATS2 & $\uparrow$ & $\uparrow$ & $\mathrm{n} / \mathrm{d}$ & $\mathrm{n} / \mathrm{d}$ & $\mathrm{n} / \mathrm{d}$ & [49] \\
\hline miR-423 & $\mathrm{n} / \mathrm{d}$ & $\uparrow$ & $\uparrow$ & $\mathrm{n} / \mathrm{d}$ & $\mathrm{n} / \mathrm{d}$ & $\mathrm{n} / \mathrm{d}$ & [153] \\
\hline miR-486-5p & MARK1 & $\uparrow$ & $\uparrow$ & $\mathrm{n} / \mathrm{d}$ & $\mathrm{n} / \mathrm{d}$ & $\mathrm{n} / \mathrm{d}$ & [112] \\
\hline miR-494-3p & PTEN & $\uparrow$ & $\uparrow$ & $\mathrm{n} / \mathrm{d}$ & $\uparrow$ & $\mathrm{n} / \mathrm{d}$ & [117] \\
\hline miR-522 & MAOB & $\uparrow$ & $\uparrow$ & $\mathrm{n} / \mathrm{d}$ & $\mathrm{n} / \mathrm{d}$ & $\mathrm{n} / \mathrm{d}$ & [132] \\
\hline miR-544a & RECK & $\uparrow$ & $\uparrow$ & $\mathrm{n} / \mathrm{d}$ & $\uparrow$ & $\mathrm{n} / \mathrm{d}$ & [111] \\
\hline miR-652 & RORA & $\uparrow$ & $\uparrow$ & $\mathrm{n} / \mathrm{d}$ & $\mathrm{n} / \mathrm{d}$ & $\uparrow$ & [72] \\
\hline miR-940 & MRVI1 & $\uparrow$ & $\uparrow$ & $\mathrm{n} / \mathrm{d}$ & $\mathrm{n} / \mathrm{d}$ & $\mathrm{n} / \mathrm{d}$ & [115] \\
\hline miR-1202 & $\mathrm{n} / \mathrm{d}$ & $\uparrow$ & $\uparrow$ & $\mathrm{n} / \mathrm{d}$ & $\mathrm{n} / \mathrm{d}$ & $\mathrm{n} / \mathrm{d}$ & [23] \\
\hline
\end{tabular}

1 —direct binding confirmed by luciferase assay, $\downarrow$-downregulated in endometrial cancer compared to normal cells, $\uparrow$ —upregulated in endometrial cancer compared to normal cells, n/d—no data, EMT—epithelial-mesenchymal transition. * - inconsistent data of miRNAs role.

We categorized miRNAs' targets into 15 groups based on their biological function in cancer (Figure 4). Tumor suppressor miRNAs that inhibit cell invasiveness and migration and were downregulated in EC (Table 2) were found to target regulators of EMT (5 targets, 12 miRNAs), growth factor signaling (14 targets, 18 miRNAs), cell cycle (9 targets, 10 miR- 
NAs), cytoskeleton (10 targets, 10 miRNAs), hormone signaling (3 targets, 4 miRNAs), phosphatidylinositol 3-kinase/protein kinase B (PI3K/AKT) signaling (3 targets, 5 miRNAs), epigenetic regulators (10 targets, 16 miRNAs). Moreover, tumor suppressor miRNAs were identified to target regulators of signaling pathways ( 7 targets, 7 miRNAs), extracellular matrix (ECM) remodeling ( 2 targets, 2 miRNAs), adhesion molecules ( 3 targets, 3 miRNAs), angiogenesis pathway ( 2 targets, 3 miRNAs), Janus kinase/signal transducers and activators of transcription (JAK-STAT) signaling pathway (1 target, 3 miRNAs), apoptosis (2 targets, 2 miRNAs), cyclic adenosine monophosphate (cAMP) signaling (1 target, 2 miRNAs), and various other mRNAs ( 7 targets, 9 miRNAs). On the contrary, oncomiRNAs that were upregulated in EC target regulators of EMT (4 targets, 5 miRNAs), hormone signaling ( 3 targets, 4 miRNAs), ECM remodeling ( 3 targets, 3 miRNAs), PI3K/AKT signaling ( 2 targets, 6 miRNAs), cell cycle ( 2 targets, 2 miRNAs), cytoskeleton regulators ( 1 target, 1 miRNA), apoptosis (1 target, 1 miRNA), and others ( 8 targets, 8 miRNAs).

\section{Pathways regulated by invasion-associated miRNA}

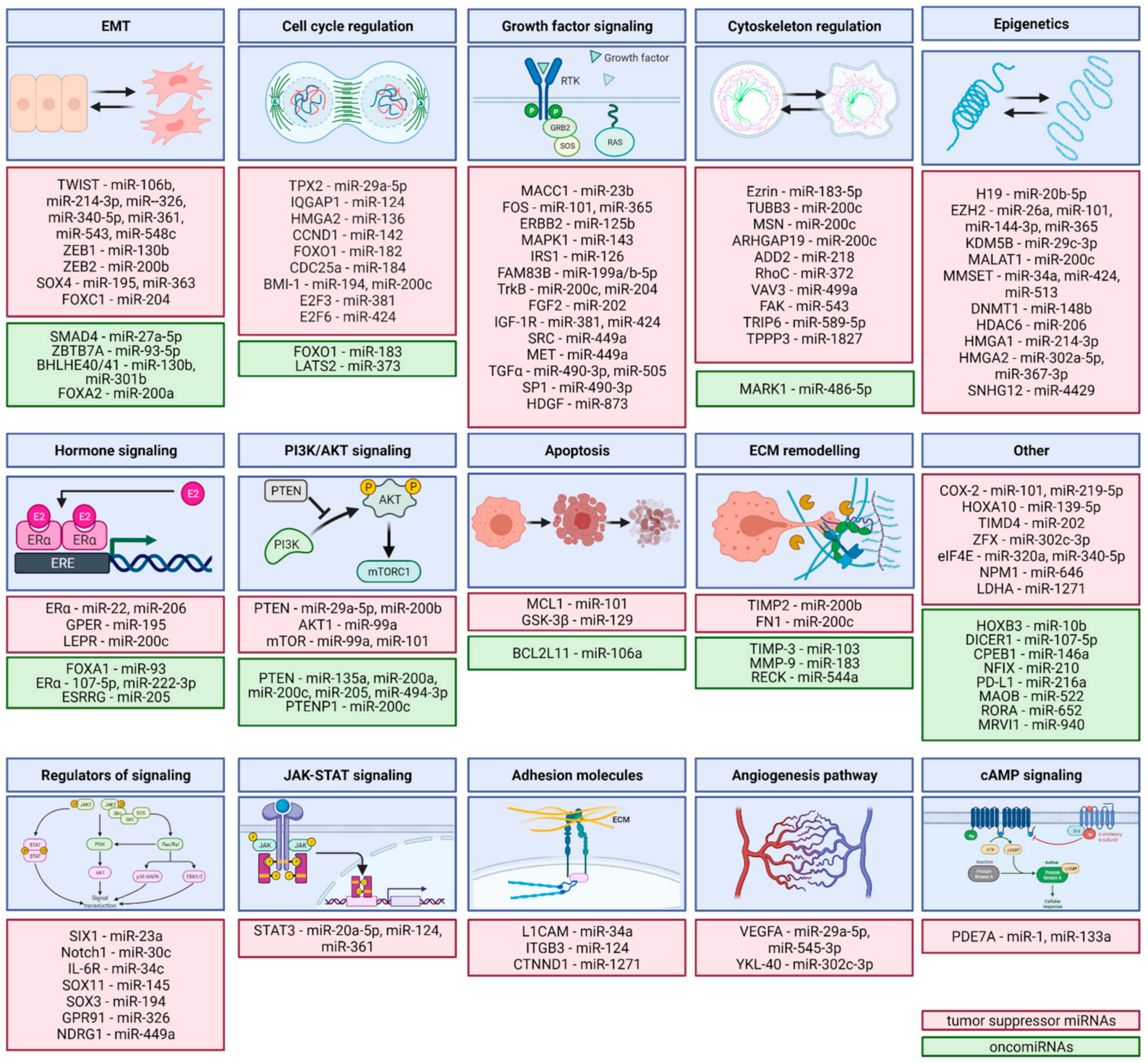

Figure 4. Direct targets of invasion-regulating miRNAs. cAMP-Cyclic Adenosine Monophosphate, EMT-epithelialmesenchymal transition, ECM-extracellular matrix, JAK-STAT_Janus Kinase/Signal Transducers and Activators of Transcription, PI3K/AKT_Phosphatidylinositol 3-Kinase/Protein Kinase B. Figure was created using Biorender.com. 


\subsection{Relationship between miRNA Expression and Clinical Parameters}

Further, we collected data from articles investigating the association between the expression of invasion-related miRNAs in tumor tissue and clinical parameters, including OS, DFS, PFS, FIGO stage, histological grade, myometrial invasion, and lymph node metastases. We identified 51 articles that correlated upregulated expression of $25 \mathrm{miR}$ NAs and downregulated expression of 41 miRNAs with at least one clinical parameter (Table 4) $[18,19,24,25,28,29,32,34,37,38,41,44,49,51,52,56-58,60,69-71,76-79,81,84,95,99,100$, $108,110,111,115,117,120,122,130-132,147,154,160,175-181]$.

Table 4. The correlation of miRNA expression and clinical parameters.

\begin{tabular}{|c|c|c|c|c|c|c|c|c|}
\hline MiRNA & Expression & OS & $\begin{array}{c}\text { DFS or } \\
\text { PFS }\end{array}$ & $\begin{array}{l}\text { FIGO } \\
\text { Stage }\end{array}$ & $\begin{array}{c}\text { Histological } \\
\text { Grade }\end{array}$ & $\begin{array}{l}\text { Myometrial } \\
\text { Invasion }\end{array}$ & $\begin{array}{l}\text { Lymph Node } \\
\text { Metastases }\end{array}$ & Ref. \\
\hline miR-10a & Upregulation & $\mathrm{n} / \mathrm{d}$ & $\mathrm{n} / \mathrm{d}$ & $\mathrm{n} / \mathrm{d}$ & $\mathrm{n} / \mathrm{d}$ & $\mathrm{n} / \mathrm{d}$ & 1 & [29] \\
\hline miR-27a & Upregulation & $\mathrm{n} / \mathrm{d}$ & $\mathrm{n} / \mathrm{d}$ & & $\mathrm{n} / \mathrm{d}$ & $\mathrm{n} / \mathrm{d}$ & $\mathrm{n} / \mathrm{d}$ & {$[60]$} \\
\hline $\operatorname{miR}-30 c-3 p$ & Upregulation & $\mathrm{n} / \mathrm{d}$ & $\mathrm{n} / \mathrm{d}$ & $\mathrm{n} / \mathrm{d}$ & $\mathrm{n} / \mathrm{d}$ & $\mathrm{n} / \mathrm{d}$ & $\uparrow a$ & [178] \\
\hline $\operatorname{miR}-34 a^{*}$ & Upregulation & $\mathrm{n} / \mathrm{d}$ & $\mathrm{n} / \mathrm{d}$ & $\mathrm{n} / \mathrm{d}$ & $\mathrm{n} / \mathrm{d}$ & $\mathrm{n} / \mathrm{d}$ & & [29] \\
\hline miR-93-5p & Upregulation & $\downarrow$ & $\mathrm{n} / \mathrm{d}$ & & $\mathrm{n} / \mathrm{d}$ & $\mathrm{n} / \mathrm{d}$ & & [95] \\
\hline miR-95 & Upregulation & $\downarrow$ & $\mathrm{n} / \mathrm{d}$ & & & $\mathrm{n} / \mathrm{d}$ & & {$[29,110]$} \\
\hline $\operatorname{miR}-107-5 p$ & Upregulation & $\mathrm{n} / \mathrm{d}$ & $\mathrm{n} / \mathrm{d}$ & & $\mathrm{n} / \mathrm{d}$ & & & [19] \\
\hline $\mathrm{miR}-130 \mathrm{~b}$ & Upregulation & & $\mathrm{n} / \mathrm{d}$ & & $\mathrm{n} / \mathrm{d}$ & $\uparrow$ & $\mathrm{n} / \mathrm{d}$ & {$[32,122]$} \\
\hline miR-181a & Upregulation & $\mathrm{n} / \mathrm{d}$ & $\mathrm{n} / \mathrm{d}$ & $\uparrow$ & $\mathrm{n} / \mathrm{d}$ & $\mathrm{n} / \mathrm{d}$ & $\mathrm{n} / \mathrm{d}$ & [37] \\
\hline miR-192 & Upregulation & $\mathrm{n} / \mathrm{d}$ & $\mathrm{n} / \mathrm{d}$ & $\mathrm{n} / \mathrm{d}$ & $\mathrm{n} / \mathrm{d}$ & $\mathrm{n} / \mathrm{d}$ & $\uparrow \mathrm{a}$ & [178] \\
\hline miR-194 & Upregulation & $\mathrm{n} / \mathrm{d}$ & $\mathrm{n} / \mathrm{d}$ & $\mathrm{n} / \mathrm{d}$ & $\mathrm{n} / \mathrm{d}$ & $\mathrm{n} / \mathrm{d}$ & $\uparrow a$ & [178] \\
\hline miR-199a & Upregulation & $\uparrow$ & $\uparrow$ & $\mathrm{n} / \mathrm{d}$ & $\mathrm{n} / \mathrm{d}$ & $\mathrm{n} / \mathrm{d}$ & $\mathrm{n} / \mathrm{d}$ & [177] \\
\hline miR-200a & Upregulation & $\mathrm{n} / \mathrm{d}$ & $\mathrm{n} / \mathrm{d}$ & & $\mathrm{n} / \mathrm{d}$ & $\mathrm{n} / \mathrm{d}$ & $\mathrm{n} / \mathrm{d}$ & [29] \\
\hline miR-203 & Upregulation & $\mathrm{n} / \mathrm{d}$ & $\mathrm{n} / \mathrm{d}$ & $\mathrm{n} / \mathrm{d}$ & $\mathrm{n} / \mathrm{d}$ & $\mathrm{n} / \mathrm{d}$ & $\uparrow a$ & [178] \\
\hline miR-205 & Upregulation & $\downarrow$ & $\mathrm{n} / \mathrm{d}$ & & $\mathrm{n} / \mathrm{d}$ & $\mathrm{n} / \mathrm{d}$ & $\mathrm{n} / \mathrm{d}$ & [29] \\
\hline miR-210 & Upregulation & $\mathrm{n} / \mathrm{d}$ & $\mathrm{n} / \mathrm{d}$ & & $\uparrow$ & $\mathrm{n} / \mathrm{d}$ & $\uparrow$ & {$[100,178]$} \\
\hline $\operatorname{miR}-222-3 p$ & Upregulation & $\mathrm{n} / \mathrm{d}$ & $\mathrm{n} / \mathrm{d}$ & & $\uparrow$ & $\mathrm{n} / \mathrm{d}$ & $\uparrow$ & [52] \\
\hline miR-301 & Upregulation & $\downarrow$ & $\mathrm{n} / \mathrm{d}$ & & $\uparrow$ & $\uparrow$ & $\uparrow$ & {$[57,178]$} \\
\hline miR-345 & Upregulation & $\mathrm{n} / \mathrm{d}$ & $\mathrm{n} / \mathrm{d}$ & $\mathrm{n} / \mathrm{d}$ & $\mathrm{n} / \mathrm{d}$ & $\mathrm{n} / \mathrm{d}$ & $\uparrow a$ & [178] \\
\hline miR-373 & Upregulation & $\downarrow$ & $\mathrm{n} / \mathrm{d}$ & & $\uparrow$ & $\uparrow$ & $\uparrow$ & {$[49,99]$} \\
\hline miR-494-3p & Upregulation & $\downarrow$ & $\mathrm{n} / \mathrm{d}$ & $\mathrm{n} / \mathrm{d}$ & $\mathrm{n} / \mathrm{d}$ & $\mathrm{n} / \mathrm{d}$ & $\mathrm{n} / \mathrm{d}$ & [117] \\
\hline miR-499 & Upregulation & $\mathrm{n} / \mathrm{d}$ & $\mathrm{n} / \mathrm{d}$ & & $\uparrow$ & $\mathrm{n} / \mathrm{d}$ & $\mathrm{n} / \mathrm{d}$ & [78] \\
\hline $\operatorname{miR}-522$ & Upregulation & $\downarrow$ & $\mathrm{n} / \mathrm{d}$ & $\mathrm{n} / \mathrm{d}$ & $\uparrow$ & $\mathrm{n} / \mathrm{d}$ & $\mathrm{n} / \mathrm{d}$ & [132] \\
\hline $\mathrm{miR}-544 \mathrm{a}$ & Upregulation & $\downarrow$ & $\mathrm{n} / \mathrm{d}$ & $\mathrm{n} / \mathrm{d}$ & $\mathrm{n} / \mathrm{d}$ & $\mathrm{n} / \mathrm{d}$ & $\mathrm{n} / \mathrm{d}$ & [111] \\
\hline miR-940 & Upregulation & $\downarrow$ & $\mathrm{n} / \mathrm{d}$ & $\mathrm{n} / \mathrm{d}$ & $\uparrow$ & $\mathrm{n} / \mathrm{d}$ & $\mathrm{n} / \mathrm{d}$ & [115] \\
\hline miR-10b & Downregulation & $\downarrow$ & $\mathrm{n} / \mathrm{d}$ & $\mathrm{n} / \mathrm{d}$ & $\mathrm{n} / \mathrm{d}$ & $\mathrm{n} / \mathrm{d}$ & $\mathrm{n} / \mathrm{d}$ & [38] \\
\hline miR-29b & Downregulation & $\downarrow$ & $\downarrow$ & & $\mathrm{n} / \mathrm{d}$ & $\mathrm{n} / \mathrm{d}$ & & {$[24,38,81]$} \\
\hline miR-29a-5p & Downregulation & $\mathrm{n} / \mathrm{d}$ & $\mathrm{n} / \mathrm{d}$ & & $\uparrow$ & $\uparrow$ & & [44] \\
\hline $\operatorname{miR}-29 c$ & Downregulation & $\downarrow$ & $\mathrm{n} / \mathrm{d}$ & $\mathrm{n} / \mathrm{d}$ & $\mathrm{n} / \mathrm{d}$ & $\mathrm{n} / \mathrm{d}$ & $\mathrm{n} / \mathrm{d}$ & [154] \\
\hline $\mathrm{miR}-34 \mathrm{a}$ * & Downregulation & $\downarrow$ & $\mathrm{n} / \mathrm{d}$ & & $\uparrow$ & $\uparrow$ & & {$[34,175]$} \\
\hline miR-34b-5p & Downregulation & $\mathrm{n} / \mathrm{d}$ & $\mathrm{n} / \mathrm{d}$ & $\mathrm{n} / \mathrm{d}$ & $\mathrm{n} / \mathrm{d}$ & $\mathrm{n} / \mathrm{d}$ & 1 & [176] \\
\hline miR-34c-3p & Downregulation & $\mathrm{n} / \mathrm{d}$ & $\mathrm{n} / \mathrm{d}$ & $\mathrm{n} / \mathrm{d}$ & $\mathrm{n} / \mathrm{d}$ & $\mathrm{n} / \mathrm{d}$ & & [176] \\
\hline miR-34c-5p & Downregulation & $\mathrm{n} / \mathrm{d}$ & $\mathrm{n} / \mathrm{d}$ & $\mathrm{n} / \mathrm{d}$ & $\mathrm{n} / \mathrm{d}$ & $\mathrm{n} / \mathrm{d}$ & 1 & [176] \\
\hline miR-100 & Downregulation & $\downarrow$ & $\mathrm{n} / \mathrm{d}$ & $\mathrm{n} / \mathrm{d}$ & $\mathrm{n} / \mathrm{d}$ & $\mathrm{n} / \mathrm{d}$ & $\mathrm{n} / \mathrm{d}$ & [76] \\
\hline miR-101 & Downregulation & & $\mathrm{n} / \mathrm{d}$ & $\mathrm{n} / \mathrm{d}$ & & 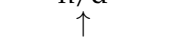 & $\mathrm{n} / \mathrm{d}$ & {$[38,56,175]$} \\
\hline miR-124 & Downregulation & $\downarrow$ & $\mathrm{n} / \mathrm{d}$ & & $\uparrow$ & $\uparrow$ & $\uparrow$ & [99] \\
\hline miR-125b & Downregulation & $\mathrm{n} / \mathrm{d}$ & $\mathrm{n} / \mathrm{d}$ & $\mathrm{n} / \mathrm{d}$ & & $\uparrow$ & $\mathrm{n} / \mathrm{d}$ & [175] \\
\hline miR-126 & Downregulation & $\mathrm{n} / \mathrm{d}$ & $\mathrm{n} / \mathrm{d}$ & & $\uparrow$ & $\mathrm{n} / \mathrm{d}$ & $\mathrm{n} / \mathrm{d}$ & [120] \\
\hline miR-139-5p & Downregulation & $\downarrow$ & $\mathrm{n} / \mathrm{d}$ & $\mathrm{n} / \mathrm{d}$ & $\mathrm{n} / \mathrm{d}$ & $\mathrm{n} / \mathrm{d}$ & $\mathrm{n} / \mathrm{d}$ & [38] \\
\hline miR-141 & Downregulation & $\downarrow$ & $\downarrow$ & $\mathrm{n} / \mathrm{d}$ & $\mathrm{n} / \mathrm{d}$ & $\mathrm{n} / \mathrm{d}$ & $\mathrm{n} / \mathrm{d}$ & [77] \\
\hline miR-142 & Downregulation & $\downarrow$ & $\mathrm{n} / \mathrm{d}$ & $\mathrm{n} / \mathrm{d}$ & $\uparrow$ & $\uparrow$ & $\mathrm{n} / \mathrm{d}$ & {$[69,175]$} \\
\hline miR-152 & Downregulation & $\downarrow$ & $\downarrow$ & $\mathrm{n} / \mathrm{d}$ & $\mathrm{n} / \mathrm{d}$ & $\mathrm{n} / \mathrm{d}$ & $\mathrm{n} / \mathrm{d}$ & [38] \\
\hline miR-184 & Downregulation & $\downarrow$ & $\mathrm{n} / \mathrm{d}$ & $\mathrm{n} / \mathrm{d}$ & $\mathrm{n} / \mathrm{d}$ & $\mathrm{n} / \mathrm{d}$ & $\uparrow$ & {$[28,176]$} \\
\hline miR-194 & Downregulation & $\downarrow$ & $\mathrm{n} / \mathrm{d}$ & $\uparrow$ & $\mathrm{n} / \mathrm{d}$ & $\mathrm{n} / \mathrm{d}$ & $\mathrm{n} / \mathrm{d}$ & [180] \\
\hline miR-195 & Downregulation & $\mathrm{n} / \mathrm{d}$ & $\mathrm{n} / \mathrm{d}$ & & $\mathrm{n} / \mathrm{d}$ & $\mathrm{n} / \mathrm{d}$ & $\uparrow a$ & [108] \\
\hline miR-202 & Downregulation & $\downarrow$ & $\mathrm{n} / \mathrm{d}$ & $\uparrow$ & $\mathrm{n} / \mathrm{d}$ & $\mathrm{n} / \mathrm{d}$ & $\uparrow$ & [25] \\
\hline miR-203 & Downregulation & $\downarrow$ & $\downarrow$ & $\mathrm{n} / \mathrm{d}$ & $\mathrm{n} / \mathrm{d}$ & $\mathrm{n} / \mathrm{d}$ & $\mathrm{n} / \mathrm{d}$ & [77] \\
\hline $\operatorname{miR}-204$ & Downregulation & $\mathrm{n} / \mathrm{d}$ & $\mathrm{n} / \mathrm{d}$ & $\uparrow$ & $\mathrm{n} / \mathrm{d}$ & $\mathrm{n} / \mathrm{d}$ & $\uparrow$ & [18] \\
\hline $\mathrm{miR}-301 \mathrm{~b}$ & Downregulation & $\downarrow$ & $\downarrow$ & $\mathrm{n} / \mathrm{d}$ & $\mathrm{n} / \mathrm{d}$ & $\mathrm{n} / \mathrm{d}$ & $\mathrm{n} / \mathrm{d}$ & [77] \\
\hline $\operatorname{miR}-361$ & Downregulation & $\mathrm{n} / \mathrm{d}$ & $\mathrm{n} / \mathrm{d}$ & $\mathrm{n} / \mathrm{d}$ & $\uparrow$ & $\mathrm{n} / \mathrm{d}$ & $\mathrm{n} / \mathrm{d}$ & [41] \\
\hline miR-372 & Downregulation & $\mathrm{n} / \mathrm{d}$ & $\mathrm{n} / \mathrm{d}$ & $\mathrm{n} / \mathrm{d}$ & $\mathrm{n} / \mathrm{d}$ & $\mathrm{n} / \mathrm{d}$ & $\uparrow$ & [130] \\
\hline $\mathrm{miR}-375$ & Downregulation & $\mathrm{n} / \mathrm{d}$ & $\mathrm{n} / \mathrm{d}$ & $\mathrm{n} / \mathrm{d}$ & $\mathrm{n} / \mathrm{d}$ & $\mathrm{n} / \mathrm{d}$ & $\uparrow$ & [176] \\
\hline miR-381 & Downregulation & $\mathrm{n} / \mathrm{d}$ & $\mathrm{n} / \mathrm{d}$ & $\uparrow$ & $\mathrm{n} / \mathrm{d}$ & $\uparrow$ & $\uparrow$ & [79] \\
\hline
\end{tabular}


Table 4. Cont

\begin{tabular}{|c|c|c|c|c|c|c|c|c|}
\hline MiRNA & Expression & OS & $\begin{array}{l}\text { DFS or } \\
\text { PFS }\end{array}$ & $\begin{array}{l}\text { FIGO } \\
\text { Stage }\end{array}$ & $\begin{array}{c}\text { Histological } \\
\text { Grade }\end{array}$ & $\begin{array}{c}\text { Myometrial } \\
\text { Invasion }\end{array}$ & $\begin{array}{l}\text { Lymph Node } \\
\text { Metastases }\end{array}$ & Ref. \\
\hline miR-424 & Downregulation & $\downarrow$ & $\mathrm{n} / \mathrm{d}$ & $\uparrow$ & $\uparrow$ & $\mathrm{n} / \mathrm{d}$ & $\uparrow$ & {$[34,58]$} \\
\hline $\operatorname{miR}-429$ & Downregulation & $\mathrm{n} / \mathrm{d}$ & $\downarrow$ & $\mathrm{n} / \mathrm{d}$ & $\mathrm{n} / \mathrm{d}$ & $\mathrm{n} / \mathrm{d}$ & $\mathrm{n} / \mathrm{d}$ & [77] \\
\hline miR-449a & Downregulation & 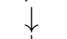 & $\mathrm{n} / \mathrm{d}$ & & 政 & $\mathrm{n} / \mathrm{d}$ & $\uparrow$ & {$[147,160]$} \\
\hline miR-455-5p & Downregulation & 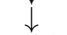 & $\downarrow$ & $\mathrm{n} / \mathrm{d}$ & $\mathrm{n} / \mathrm{d}$ & $\mathrm{n} / \mathrm{d}$ & $\mathrm{n} / \mathrm{d}$ & [38] \\
\hline miR-490-3p & Downregulation & $\mathrm{n} / \mathrm{d}$ & $\mathrm{n} / \mathrm{d}$ & $\mathrm{n} / \mathrm{d}$ & $\mathrm{n} / \mathrm{d}$ & $\uparrow$ & $\uparrow$ & {$[70]$} \\
\hline $\operatorname{miR}-497^{1}$ & Downregulation & $\downarrow$ & $\mathrm{n} / \mathrm{d}$ & & $\uparrow$ & $\mathrm{n} / \mathrm{d}$ & $\mathrm{n} / \mathrm{d}$ & [179] \\
\hline miR-505 & Downregulation & $\mathrm{n} / \mathrm{d}$ & $\mathrm{n} / \mathrm{d}$ & 1 & $\mathrm{n} / \mathrm{d}$ & $\mathrm{n} / \mathrm{d}$ & $\uparrow$ & [131] \\
\hline miR-513 & Downregulation & $\downarrow$ & $\mathrm{n} / \mathrm{d}$ & $\mathrm{n} / \mathrm{d}$ & $\mathrm{n} / \mathrm{d}$ & $\mathrm{n} / \mathrm{d}$ & $\mathrm{n} / \mathrm{d}$ & [34] \\
\hline miR-548c & Downregulation & $\downarrow$ & $\mathrm{n} / \mathrm{d}$ & $\mathrm{n} / \mathrm{d}$ & $\mathrm{n} / \mathrm{d}$ & $\mathrm{n} / \mathrm{d}$ & $\mathrm{n} / \mathrm{d}$ & [71] \\
\hline miR-873 & Downregulation & $\mathrm{n} / \mathrm{d}$ & $\mathrm{n} / \mathrm{d}$ & & $\uparrow$ & $\mathrm{n} / \mathrm{d}$ & $\mathrm{n} / \mathrm{d}$ & [84] \\
\hline miR-99a & Downregulation & $\mathrm{n} / \mathrm{d}$ & $\mathrm{n} / \mathrm{d}$ & $\uparrow$ & $\uparrow$ & $\mathrm{n} / \mathrm{d}$ & $\mathrm{n} / \mathrm{d}$ & [51] \\
\hline miR-200 family & Downregulation & $\mathrm{n} / \mathrm{d}$ & $\mathrm{n} / \mathrm{d}$ & $\mathrm{n} / \mathrm{d}$ & $\uparrow$ & $\mathrm{n} / \mathrm{d}$ & $\mathrm{n} / \mathrm{d}$ & [181] \\
\hline miR-1228 & Downregulation & $\mathrm{n} / \mathrm{d}$ & $\downarrow$ & $\mathrm{n} / \mathrm{d}$ & $\mathrm{n} / \mathrm{d}$ & $\mathrm{n} / \mathrm{d}$ & $\mathrm{n} / \mathrm{d}$ & {$[77]$} \\
\hline
\end{tabular}

$\downarrow$-decreased, $\uparrow$-increased, $\mathrm{n} / \mathrm{d}$-no data, a-lympho-vascular invasion, ${ }^{*}$-inconsistent data of miRNAs expression and clinical parameters, OS—overall survival, DFS—disease-free survival, PFS - progression-free survival, FIGO stage-The International Federation of Gynecology and Obstetrics staging classification.

Increased levels of nine miRNAs, miR-93-5p, miR-95, miR-205, miR-301, miR-373, miR-494-3p, miR-522, miR-544a and miR-940 in tumor tissue as well as decreased levels of 22 miRNAs, miR-10b, miR-29b, miR-29c, miR-34a, miR-100, miR-101, miR-124, miR-139-5p, miR-141, miR-142, miR-152, miR-184, miR-194, miR-202, miR-203, miR-301b, miR-424, miR-449a, miR-455-5p, miR-497, miR-513, and miR-548c were correlated with a shorter OS (Table 4). On the contrary, upregulated expression of miR-130b and miR-199a was associated with a longer OS in ECs. Upregulation of four miRNAs, miR-107-5p, miR-130b, miR-301, miR-373 as well as downregulation of eight miRNAs, miR-29a-5p, miR-34a, miR101, miR-124, miR-125b, miR-142, miR-381, miR-490-3p, were associated with the invasion of the myometrium. We identified nine upregulated miRNAs and 17 downregulated miRNAs that correlated with lymph node metastases. Moreover, an increase of miR-30c-3p, miR-192, miR-194, miR-203, miR-345 and a decrease of miR-195 were associated with lymphovascular invasion (Table 4).

\section{Discussion}

Tumor expansion and progression are enabled by coordinated dysregulation of various mechanisms. The process of tumor invasion and metastasis is composed of several steps, including primary tumor growth, migration, local invasion, intravasation, survival in the circulation, extravasation, and pre-metastatic niche formation. All of these steps are regulated by a variety of different miRNAs [12].

In this work, we provide a comprehensive overview of miRNAs that are dysregulated in EC and contribute to tumor progression. We identified 106 dysregulated miRNAs through a systematic literature review. Small RNA-seq analysis revealed that 239 out of 359 detected miRNAs are dysregulated in EC compared to healthy adjacent endometrial tissue [182]. Further, analysis of miRNA-seq data from The Cancer Genome Atlas (TCGA) database expanded the list of dysregulated miRNAs to 531 [179]. Previous systematic reviews identified, respectively, 106 miRNAs [183], 261 miRNAs [184], 310 miRNAs [185] dysregulated in ECs. However, these studies included also many miRNAs detected by high throughput methods, including microarrays and small RNA-seq, without confirmation by the RT-qPCR method that is a gold standard for miRNAs analysis [186]. Moreover, in contrast to previous reviews, we included only miRNAs with a confirmed role in the regulation of EC cell migration, invasiveness, and metastasis in vitro and/or in vivo.

\subsection{Regulatory Network of Invasion-Associated miRNAs}

MiRNAs orchestrate tumor invasion and metastasis by targeting various mRNAs. Complex regulation of multiple signaling pathways, including PI3K/AKT [187], and cellular processes, including EMT and cytoskeleton remodeling [188], by miRNAs enable the control of the invasiveness and metastasis of cancer cells. We classified targets of miRNAs into 15 categories based on their function. Notably, multiple miRNAs target 
regulators of multiple cellular processes and create a complex network of interactions. Hence, they have been assigned to a given category based on their best-described function in the EC (Figure 4).

\subsubsection{Epithelial-Mesenchymal Transition (EMT)}

One of the most important processes in the myometrial invasion of EC is an EMT [188]. EMT enables the acquisition of the mesenchymal-like features from the epithelial cells and occurs physiologically during embryonic development and tissue regeneration [189]. EMT is characterized by the loss of adherent junctions, downregulation of epithelial markers (cytokeratins and E-cadherin) but upregulation of mesenchymal markers, including $\mathrm{N}$-cadherin, vimentin, and fibronectin [12,189]. EMT is regulated by multiple signaling pathways, including Transforming Growth Factor $\beta$ (TGF- $\beta$ )/SMAD signaling, Wnt pathway, and PI3K/AKT, and is orchestrated by Snail, Slug, Smug, SRY-Box Transcription Factor 1 (SOX1), Forkhead Box C1 (FOXC1), Zinc Finger E-Box Binding Homeobox 1/Zinc Finger E-Box Binding Homeobox 2 (ZEB1/ZEB2), and Twist-related protein 1/Twist-related protein 2 (TWIST1/TWIST2) transcription factors [190,191].

We identified 20 suppressors of this process in EC cells (miR-20a-5p, miR-26a, miR-34a, miR-101, miR-106b, miR-124, miR-130b, miR-183-5p, miR-194, miR-195, miR-199a/b-5p, miR-200c, miR-202, miR-214-3p, miR-320a, miR-326, miR-340-5p, miR-365, miR-424, and $\mathrm{miR}-513$ ) and seven oncomiRNAs that stimulate cells transition (miR-93, miR-130b, miR183, miR-200a, miR-200c, miR-205, and miR-301b) (Table 3). Moreover, we identified microRNAs that target nine regulators of EMT, including TWIST, ZEB1/ZEB2, SRY-Box Transcription Factor 4 (SOX4), FOXC1, SMAD4, Zinc Finger and BTB Domain Containing 7A (ZBTB7A), Basic helix-loop-helix transcription factors e40/41 (BHLHE40/41), and Forkhead Box A2 (FOXA2). TWIST, a crucial transcription factor regulating EMT, was identified as a target of several miRNAs in this study (miR-106b, miR-214-3p, miR-326, miR-340-5p, miR-361, miR-543, and miR-548c). Similarly, ZEB1 and ZEB2 transcription factors were identified to be targets of $\mathrm{miR}-130 \mathrm{~b}$ and $\mathrm{miR}-200 \mathrm{~b}$, respectively (Figure 4 ).

\subsubsection{Cell Cycle}

The cell cycle is regulated by several protein checkpoints including cyclins, cyclindependent kinases (CDKs), and their inhibitors (CKI), that may be inactivated or mutated in cancer cells that lead to uncontrolled proliferation and thus to the progression of cancer [192,193]. There are several I-III phases clinical trials of the application of CKI in EC [194]. MiRNAs are described to control the expression of genes related to the cell cycle [195]. In this systematic review, we summarize tumor suppressor miRNAs (miR-29a-5p, miR-124, miR-136, miR-142, miR-182, miR-184, miR-194, miR-200c, miR-381, miR-424) and their targets (TPX2 Microtubule Nucleation Factor (TPX2), IQ Motif Containing GTPase Activating Protein 1 (IQGAP1), High Mobility Group AT-Hook 2 (HMGA2), C-terminal Cyclin D1 (CCND1), Forkhead Box O1 (FOXO1), Cell division cycle 25a (CDC25a), B-lymphoma Moloney murine leukemia virus insertion region-1 (BMI-1), Transcription Factor 3 (E2F3), and E2F Transcription Factor 6 (E2F6)) and oncomiRNAs (miR-183 and miR-373) and their targets (FOXO1 and Large Tumor Suppressor Kinase 2 (LATS2)) involved in cell-cycle regulation. HMGA2 is an oncogene upregulated in several cancers that stimulates proliferation and invasion. HMGA2 is targeted in ECs by tumor suppressor miR-136 [65]. CCND1 activates cyclin-dependent kinase (CDK) $4 / 6$ and thus stimulates proliferation, migration, and invasion of cells [196]. In this study, CCND1 was found to be targeted by miR-142 [69].

\subsubsection{Growth Factors and Regulators of Signaling}

Overactivation of growth factors signaling is crucial in tumor development and progression. Thus, inhibitors of signaling pathways are currently under investigation in EC patients [197]. In this study, we identified multiple growth factors and regulators of signaling that promote EC cell invasion and were targets of different miRNAs. MiRNAs (miR-23b, miR-101, miR-125b, miR-126, miR-143, miR-199a/b-5p, miR-200c, miR-202, 
miR-204, miR-365, miR-381, miR-424, miR-449a, miR-490-3p, miR-505, and miR-873) that target growth factors (Metastasis-associated in colon cancer protein 1 (MACC1), Fos ProtoOncogene (FOS), Erb-B2 Receptor Tyrosine Kinase 2 (ERBB2), Mitogen-Activated Protein Kinase (MAPK1), Insulin Receptor Substrate 1 (IRS1), Family With Sequence Similarity 83 Member B (FAM83B), Tropomyosin receptor kinase B (TrkB), Fibroblast growth factor 2 (FGF2), Insulin-like growth factor 1 (IGF-1R), SRC Proto-Oncogene (SRC), MET ProtoOncogene (MET), Transforming Growth Factor Alpha (TGF $\alpha)$, Sp1 Transcription Factor (SP1), and Hepatoma-derived Growth Factor (HDGF)) decrease migration, invasion, and EMT in vitro and tumor growth and metastases in vivo, as well as correlate with EC stage, grade, and patients outcome. TGF $\alpha$ promotes the progression of cancer by increasing proliferation and differentiation. In our study, miR-490-3p and miR-505 target TGF $\alpha$ and act as tumor suppressor miRNAs [27,70].

Additionally, several tumor-suppressing miRNAs (miR-23a, miR-30c, miR-34c, miR145, miR-194, miR-326, and miR-449a) target regulators of signaling (SIX Homeobox 1 (SIX1), Notch homolog 1 (Notch1), Interleukin 6 Receptor (IL-6R), SRY-Box Transcription Factor 11 (SOX11), SRY-Box Transcription Factor 3 (SOX3), G protein-coupled receptor 91 (GPR91), and N-Myc Downstream Regulated 1 (NDRG1)). Notch1 is associated with EMT, metastases, and poor prognosis in different cancers including EC [198,199]. MiR-30c targets Notch1 and decreases migration and invasion, however, it is associated with increased lymphovascular invasion in EC patients [96,164,178].

\subsubsection{Cytoskeleton Regulation}

The polarization of the cytoskeleton and formation of the leading protrusion initiate cell migration [200]. During this process, the cell cytoskeleton undergoes dynamic changes that are regulated by multiple factors interacting with actin microfilaments or tubulin [201]. We identified eight tumor suppressor miRNAs (miR-183-5p, miR-200c, miR-218, miR-372, miR-499a, miR-543, miR-589-5p, and miR-1827) that target regulators of the cytoskeleton (Ezrin, Tubulin Beta 3 Class III (TUBB3), moesin (MSN), Rho GTPase activating protein 19 (ARHGAP19), adducin 2 (ADD2), Ras Homolog Family Member C (RhoC), Vav Guanine Nucleotide Exchange Factor 3 (VAV3), Focal adhesion kinase (FAK), Thyroid Hormone Receptor Interactor 6 (TRIP6), Tubulin Polymerization Promoting Protein Family Member 3 (TPPP3) ) and inhibits EC cell migration and invasiveness. Ezrin, which overexpression in EC is related to poor prognosis [202], is targeted by downregulated miR-183-5p. Moreover, a well-described tumor suppressor miR-200c [203] targets TUBB3, MSN, and ARHGAP19, which regulate multiple cytoskeletal-related events. On the contrary, miR-486-5p was found to promote EC cell invasiveness by targeting Microtubule affinity regulating kinase 1 (MARK1) [112], a tumor suppressor in several types of cancer [112,204,205].

\subsubsection{Epigenetics}

Gene expression is modified by multiple epigenetic mechanisms, including DNA methylation, histone acetylation and methylation, and non-coding RNAs. Dysregulation of these mechanisms in cancer affects a variety of cellular responses [206]. We selected 16 tumor suppressor miRNAs (miR-20b, miR-26a, miR-29c-3p, miR-34a, miR-101, miR144-3p, miR-148b, miR-200c, miR-206, miR-214-3p, miR-302a-5p, miR-365, miR-367-3p, miR-424, miR-513, miR-4429) that targeted 10 regulators, including Enhancer of Zeste Homolog 2 (EZH2), Lysine Demethylase 5B (KDM5B), Multiple Myeloma SET Domain (MMSET), Metastasis Associated Lung Adenocarcinoma Transcript 1 (MALAT1), DNA methyltransferase 1 (DNMT1), Histone Deacetylase 6 (HDAC6), High Mobility Group AT-Hook 1 (HMGA1), High Mobility Group AT-Hook 2 (HMGA2), Small Nucleolar RNA Host Gene 12 (SNHG12), and H19 Imprinted Maternally Expressed Transcript (H19). $\mathrm{EZH} 2$ is the enzymatic catalytic subunit of the polycomb-repressive complex 2 (PRC2) that represses transcription of multiple genes, including tumor-suppressors [207]. It is overexpressed in many types of cancer, including EC [208], and is correlated with decreased 
DFS and OS [209]. EZH2 is targeted by miR-26a and miR-101, which are downregulated in EC $[42,46]$.

Importantly, epigenetic alterations affect miRNAs profile in EC [185]. It was found that loci of genes coding oncomiRNAs miR-130a/b, miR-182, miR-200b, miR-208a, miR-222, miR-625 are hypo-methylated in EC while genes of tumor-suppressor miRNAs miR-34b, miR-124a-1, miR-124a-2, miR-124a-3, miR-129-2, miR-137, miR-152, miR-638, miR-663 are hyper-methylated [185].

\subsubsection{Hormone Signaling}

Dysregulation of hormone signaling is one of the most important factors in EC development. From them, estrogen seems to be crucial in EC pathogenesis. Estrogen binds to estrogen receptors (ER) or G protein-coupled estrogen receptor 1 (GPER1) to regulate gene transcription and to promote cancer cell pathways [210-212]. Estrogen signaling and miRNAs are in complex interaction since estrogen modulates miRNAs expression but also miRNAs target their receptors [213]. In this study, we identified miRNAs that suppress EC progression via binding to estrogen receptors. MiR-22 and miR-206 target ER what inhibits EC cell invasion and migration [121,156,168]. Moreover, miR-195 targets GPER1 and inhibits EC migration, invasion, and EMT in vitro [139]. On the other hand, miR-107-5p and miR-222-3p targeting ERa and miR-205 targeting Estrogen Related Receptor Gamma (ESRRG) act as oncomiRNAs by enhancing migration, invasion in vitro or/and tumor growth in vivo $[19,52,68]$.

\subsubsection{Phosphatidylinositol 3-Kinase/Protein Kinase B (PI3K/AKT) Pathways}

Estrogen stimulates the PI3K/Akt pathway, one of the most important pathways responsible for EC proliferation, migration, invasion, and EMT [210]. The activation of this oncogenic pathway is regulated by PTEN, a well-known tumor suppressor potently downregulated in EC [214]. Our study highlights oncomiRNAs (miR-135a, miR-200a, miR-200c, miR-205, and miR-494-3p) and tumor suppressor miRNAs (miR-29a-5p, miR-99a, miR-101, and miR-200b) that target members of this pathway.

\subsubsection{Apoptosis}

Apoptosis is a process of programmed cell death via the activation of different pathways [215]. Novel therapies targeting apoptosis inhibit B-cell lymphoma-2 (Bcl-2), induced Myeloid Leukemia Cell Differentiation Protein 1 (MCL-1), or target the P53 pathway [216]. In this study, we identified tumor suppressor miRs and oncomiRNAs that target mRNAs of proteins involved in the regulation of apoptosis. Tumor suppressor miR-101 that targets MCL-1, a protein belonging to the Bcl-2 family that inhibits apoptosis, inhibits migration, invasion, and EMT in vitro and tumor growth in vivo, and influences patient survival. Moreover, we recognized invasion-suppressing miR-129 that targets Glycogen synthase kinase-3 $\beta$ (GSK3 $\beta$ ), a serine/threonine-protein kinase that inhibits apoptosis via NF- $k B$ activation [217]. MiR-106a targets proapoptotic Bcl-2-like protein 11 (BCL2L11) and acts as oncomiRNA and stimulates migration, invasion in vitro, and tumor growth in vivo [218].

\subsubsection{Extracellular Matrix (ECM) Remodeling}

Remodeling of ECM is crucial for a cancer cell to initiate an invasion of adjacent tissues. Among multiple factors regulating this process, the most important are Matrix Metalloproteinases (MMPs) and their inhibitors Tissue Inhibitors of Metalloproteinases (TIMPs). MMPs degrade collagen, fibrinogen, fibronectin (FN1), and others and in this way enable tumor cells to invade [12]. In this study, miR-200b targets TIMP-2, miR-200c targets FN1 and both miRNAs act as tumor suppressor miRNAs. On the other hand, miR-103 targets TIMP-3 and stimulates invasion. Moreover, oncomiRNA miR-183 targets MMP-9 and miR-544a targets Reversion Inducing Cysteine Rich Protein with Kazal Motifs (RECK). These results show the complexity of the regulatory processes involved in ECM remodeling. 
One target may be regulated by several miRNAs, as well as miRNAs may have a role in the regulation of invasion by different mRNAs [219].

4.1.10. Janus Kinase/Signal Transducers and Activators of Transcription (JAK-STAT) Signaling

JAK-STAT pathway is a known regulator of tumor progression [220]. JAK-STAT pathway is dysregulated in endometrial cancer cells what leads to increased proliferation [221]. In this study, we identified three miRNAs (miR-20a-5p, miR-124, and miR-361) that target STAT3. MiR-20a-5p inhibits invasion and EMT [40]. MiR-124 decreases migration, invasion, and EMT in vitro and is associated with patients' outcomes [50,99]. Conversely, miR-361 inhibits migration and invasion in vitro and tumor growth in vivo, as well as is negatively correlated with histological grade [169].

\subsubsection{Adhesion Molecules}

Tumor cell migration and invasiveness strictly depend on the cell adhesion molecules, which include cadherins, integrins, selectins, Ig-superfamily Cell Adhesion Molecules (CAMs), and others [12,222,223]. We identified three tumor suppressor miRNAs (miR34a, miR-124, and miR-1271) that target three adhesion molecules (L1 Cell Adhesion Molecule (L1CAM), Integrin Subunit Beta 3 (ITGB3), Catenin Delta 1 (CTNND1)). L1CAM is primarily a regulator of nervous system development [224]. However, further studies demonstrated its role in the regulation of tumor progression [225]. In EC patients, L1CAM is an important prognostic factor and an independent predictor of poor survival [226-228]. Upregulation of L1CAM in EC is at least in part caused by the downregulation of L1CAMtargeting tumor suppressor miR-34a.

\subsubsection{Angiogenesis}

Angiogenesis is one of the hallmarks of cancer according to Hanahan and Weinberg [14] playing a crucial role in the progression of EC. One of the most important proangiogenic factors is the Vascular Endothelial Growth Factor (VEGF) [229]. VEGF is often targeted in novel therapies including anti-VEGF antibody (bevacizumab), VEGF trap (aflibercept), or Tyrosine Kinase Inhibitors (TKI). Therapy with bevacizumab was approved by FDA in different tumors including colorectal cancer and is currently tested in EC $[229,230]$. An increased number of clinical trials are assessing the efficacy of that novel therapies in monotherapy as well as in a combination with other applied therapies but still lacks a personalized approach [229]. In this study, we found two miRNAs (miR-29a-5p and miR-545-3p) that target VEGF and thus influence EC progression.

\subsubsection{Cyclic Adenosine Monophosphate (cAMP) Signaling}

cAMP pathways are described to inhibit the invasion of cancer $[231,232]$. cAMP promotes apoptosis and decreases tumor growth [233]. The cAMP-specific phosphodiesterase 7A (PDE7A) hydrolyzes cAMP [97]. MiR-1 and miR-133a target PDE7A which leads to the inhibition of EC cell migration and invasion in vitro.

\subsection{The Role of miRNAs in EC Diagnosis and Management}

There is a great clinical interest in the determination of biomarkers for the diagnosis and management of EC, especially to enable individualized cancer care in the light of genomic classification [234]. Despite the identification of many prognostic biomarkers in EC $[235,236]$, none of them are routinely used for diagnostic or prognostic purposes. Recent systematic reviews and meta-analyses revealed the clinical utility for the use of miRNAs as biomarkers in a variety of cancer, including bladder cancer [237], prostate cancer [238], ovarian cancer [239], and breast cancer [240], which makes miRNAs promising candidate for biomarkers in EC (Figure 5). 


\section{miRNAs as diagnostic biomarkers and therapeutics}

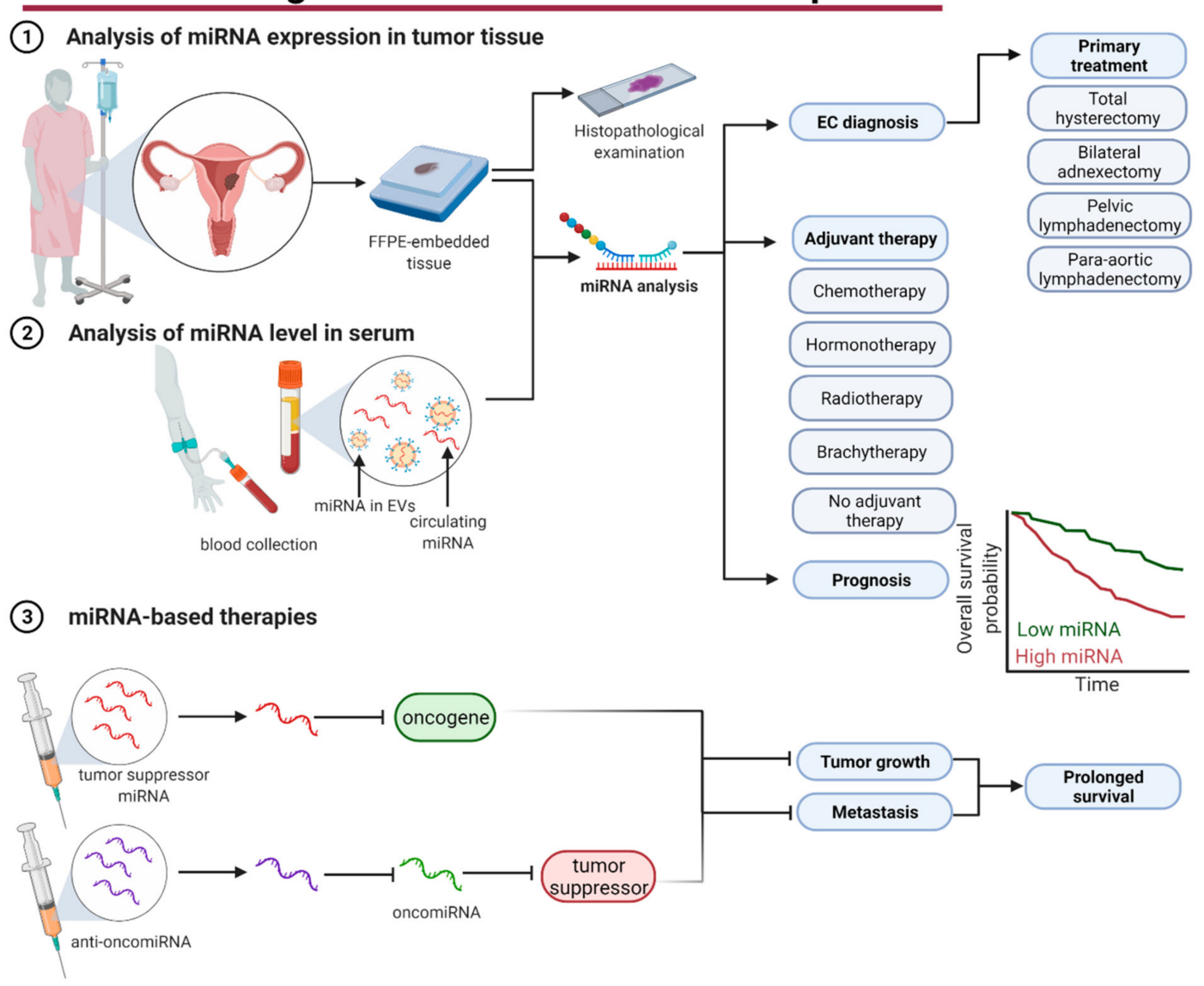

Figure 5. miRNAs as diagnostic biomarkers and therapeutics. miRNAs are promising biomarkers that can be analyzed either from tumor tissue (1) or from blood obtained from EC patients (2). miRNAs may support EC diagnosis and indicate primary and adjuvant treatment. Moreover, their expression is an important prognostic factor. miRNA-based therapies that include tumor suppressor miRNAs and anti-oncomiRNAs are currently tested in preclinical and clinical trials (3). EVs-extracellular vesicles, FFPE—formalin-fixed, paraffin-embedded. Figure was created using Biorender.com.

Importantly, dysregulation of miRNAs expression can be also detected in plasma or serum (Figure 5). It was identified that the concentration of $19 \mathrm{miRNAs}$ is increased in EC patients and the concentration of 10 miRNAs is decreased compared to healthy women [184]. Exploring publications we found that the concentration of six miRNAs was decreased and the concentration of 11 miRNAs was higher in EC patients and five from them (miR-27, miR-29b, miR-95, miR-203, and miR-449a) were associated with tumor myometrial invasion or lymph node metastases $[60,77,81,110]$.

MiRNAs are also promising therapeutics in clinical oncology. Among described in this study miRNAs regulating tumor invasiveness, the effectiveness and safety of miR-34 have been tested in clinical trials. Despite the good efficacy of MRX34 (the liposome-miR-34 complex) in intravenous administration with dexamethasone premedication, the clinical trial was terminated due to severe adverse events including deaths [241,242]. One of the challenges with using miRNAs or their inhibitors in clinical practice is finding the correct route of administration because of their toxicity, limited stability, and low penetrance to target cells. Several delivery strategies have been developed including local delivery, viral delivery, lipid-based and polymer-based vectors [243]. So far, despite numerous preclinical studies and clinical trials none of the miRNA-based therapeutics have complied with expectations [243-245].

Some limitations should be acknowledged in this systematic review. First, to minimize the rate of false-positive results, we excluded studies that did not confirm the expression of miRNAs by RT-qPCR. Thus, we excluded studies that performed only global miRNA 
profiling. It may introduce selection bias and cause exclusion of relevant miRNAs as well as limits the number of comparable results. Second, most of the studies did not distinguish types of EC in the analyzed cohort or focus on EC type I which may introduce bias. Indeed, some studies demonstrate the differences in the miRNA profile in different types of EC [246,247]. Third, the included studies present heterogeneity in sample types (fresh frozen or formalin-fixed, paraffin-embedded (FFPE) tissues), types of controls, and heterogeneity of study group. Fourth, a small number of miRNAs were assessed by at least two independent studies. Moreover, most of the studies that assessed the correlation of miRNA level and clinical parameters relied on a small group of patients and many of the included articles did not report relevant statistical parameters. Therefore, a meta-analysis could not be performed. Despite these limitations, our article is the first systematic review that comprehensively discusses data regarding the role of miRNAs in EC invasiveness and metastasis.

\section{Conclusions}

In this systematic review, we comprehensively reviewed miRNAs that are crucial regulators of EC invasiveness and metastasis. Extensive research revealed a complex regulatory network of tumor suppressors and oncomiRNAs that orchestrate tumor progression. Identified miRNAs control EC cell migration, invasion, and EMT in vitro, as well as tumor growth and lymph node metastases in vivo. These miRNAs regulate EC by targeting various members of pathways involved in diverse steps of cancer progression. That makes miRNAs promising candidates for diagnostic and prognostic biomarkers and potential therapeutic targets. Nonetheless, miRNAs that can be useful in clinical practice remain to be identified yet. Further large translational and clinical studies are needed to assess the clinical utility of miRNAs.

Author Contributions: Conceptualization, K.K., T.M.G.; writing—original draft preparation, K.K., T.M.G., A.K., A.M.; visualization, T.M.G.; writing-review and editing, T.M.G., K.K., A.K., A.M., P.K.W.; supervision, P.K.W. All authors have read and agreed to the published version of the manuscript. K.K. and T.M.G. contributed equally to this work.

Funding: This research was funded by the Medical University of Warsaw, grant number 1MN/M/MG1/N /20. The APC was funded by the Medical University of Warsaw.

Conflicts of Interest: The authors declare no conflict of interest of the study. The funders had no role in the design of the study; in the collection, analyses, or interpretation of data; in the writing of the manuscript, or in the decision to publish the results.

\section{References}

1. Siegel, R.L.; Miller, K.D.; Jemal, A. Cancer statistics, 2020. CA Cancer J. Clin. 2020, 70, 7-30. [CrossRef] [PubMed]

2. Lu, K.H.; Broaddus, R.R. Endometrial Cancer. N. Engl. J. Med. 2020, 383, 2053-2064. [CrossRef] [PubMed]

3. Rahatli, S.; Dizdar, O.; Kucukoztas, N.; Oguz, A.; Yalcin, S.; Ozen, O.; Reyhan, N.H.; Tarhan, C.; Yildiz, F.; Dursun, P.; et al. Good outcomes of patients with stage IB endometrial cancer with surgery alone. Asian Pac. J. Cancer Prev. 2014, 15, $3891-3893$. [CrossRef] [PubMed]

4. Chen, K.S.; Berhane, H.; Gill, B.S.; Olawaiye, A.; Sukumvanich, P.; Kelley, J.L.; Boisen, M.M.; Courtney-Brooks, M.; Comerci, J.T.; Edwards, R.; et al. Outcomes of stage II endometrial cancer: The UPMC Hillman Cancer Center experience. Gynecol. Oncol. 2017, 147, 315-319. [CrossRef] [PubMed]

5. Saleh, M.; Virarkar, M.; Bhosale, P.; El Sherif, S.; Javadi, S.; Faria, S.C. Endometrial Cancer, the Current International Federation of Gynecology and Obstetrics Staging System, and the Role of Imaging. J. Comput. Assist. Tomogr. 2020, 44, 714-729. [CrossRef] [PubMed]

6. Soslow, R.A.; Tornos, C.; Park, K.J.; Malpica, A.; Matias-Guiu, X.; Oliva, E.; Parkash, V.; Carlson, J.; McCluggage, W.G.; Gilks, C.B. Endometrial Carcinoma Diagnosis: Use of FIGO Grading and Genomic Subcategories in Clinical Practice: Recommendations of the International Society of Gynecological Pathologists. Int. J. Gynecol. Pathol. Off. J. Int. Soc. Gynecol. Pathol. 2019, 38, S64-S74. [CrossRef] [PubMed]

7. Mehlich, D.; Garbicz, F.; Włodarski, P.K. The emerging roles of the polycistronic miR-106b 25 cluster in cancer-A comprehensive review. Biomed. Pharmacother. 2018, 107, 1183-1195. [CrossRef]

8. Williams, M.; Cheng, Y.Y.; Blenkiron, C.; Reid, G. Exploring Mechanisms of MicroRNA Downregulation in Cancer. MicroRNA 2017, 6, 2-16. [CrossRef] 
9. Peng, Y.; Croce, C.M. The role of MicroRNAs in human cancer. Signal. Transduct. Target. Ther. 2016, 1, 15004. [CrossRef]

10. Pełka, K.; Klicka, K.; Grzywa, T.M.; Gondek, A.; Marczewska, J.M.; Garbicz, F.; Szczepaniak, K.; Paskal, W.; Włodarski, P.K. miR-96-5p, miR-134-5p, miR-181b-5p and miR-200b-3p heterogenous expression in sites of prostate cancer versus benign prostate hyperplasia-archival samples study. Histochem. Cell Biol. 2021, 155, 423-433. [CrossRef]

11. Vedanayagam, J.; Chatila, W.K.; Aksoy, B.A.; Majumdar, S.; Skanderup, A.J.; Demir, E.; Schultz, N.; Sander, C.; Lai, E.C. Cancerassociated mutations in DICER1 RNase IIIa and IIIb domains exert similar effects on miRNA biogenesis. Nat. Commun. 2019, 10, 3682. [CrossRef]

12. Grzywa, T.M.; Klicka, K.; Włodarski, P.K. Regulators at Every Step-How microRNAs Drive Tumor Cell Invasiveness and Metastasis. Cancers 2020, 12, 3709. [CrossRef]

13. Grzywa, T.M.; Klicka, K.; Rak, B.; Mehlich, D.; Garbicz, F.; Zieliński, G.; Maksymowicz, M.; Sajjad, E.; Włodarski, P.K. Lineagedependent role of miR-410-3p as oncomiR in gonadotroph and corticotroph pituitary adenomas or tumor suppressor miR in somatotroph adenomas via MAPK, PTEN/AKT, and STAT3 signaling pathways. Endocrine 2019, 65, 646-655. [CrossRef]

14. Hanahan, D.; Weinberg, R.A. Hallmarks of Cancer: The Next Generation. Cell 2011, 144, 646-674. [CrossRef]

15. Ghafouri-Fard, S.; Shoorei, H.; Anamag, F.T.; Taheri, M. The Role of Non-Coding RNAs in Controlling Cell Cycle Related Proteins in Cancer Cells. Front. Oncol. 2020, 10. [CrossRef]

16. Leone, P.; Buonavoglia, A.; Fasano, R.; Solimando, A.G.; De Re, V.; Cicco, S.; Vacca, A.; Racanelli, V. Insights into the Regulation of Tumor Angiogenesis by Micro-RNAs. J. Clin. Med. 2019, 8, 2030. [CrossRef]

17. Si, W.; Shen, J.; Zheng, H.; Fan, W. The role and mechanisms of action of microRNAs in cancer drug resistance. Clin. Epigenetics 2019, 11, 25. [CrossRef]

18. Bao, W.; Wang, H.H.; Tian, F.J.; He, X.Y.; Qiu, M.T.; Wang, J.Y.; Zhang, H.J.; Wang, L.H.; Wan, X.P. A TrkB-STAT3-miR-204-5p regulatory circuitry controls proliferation and invasion of endometrial carcinoma cells. Mol. Cancer 2013, 12, 155. [CrossRef]

19. Bao, W.; Zhang, Y.; Li, S.; Fan, Q.; Qiu, M.; Wang, Y.; Li, Y.; Ji, X.; Yang, Y.; Sang, Z.; et al. miR-107-5p promotes tumor proliferation and invasion by targeting estrogen receptor- $\alpha$ in endometrial carcinoma. Oncol. Rep. 2019, 41, 1575-1585. [CrossRef]

20. Bing, L.; Hong, C.; Li-Xin, S.; Wei, G. MicroRNA-543 suppresses endometrial cancer oncogenicity via targeting FAK and TWIST1 expression. Arch. Gynecol. Obstet. 2014, 290, 533-541. [CrossRef]

21. Chang, L.; Yuan, Z.; Shi, H.; Bian, Y.; Guo, R. miR-145 targets the SOX11 3'UTR to suppress endometrial cancer growth. Am. J. Cancer Res. 2017, 7, 2305-2317. [PubMed]

22. Chang, L.; Zhang, D.; Shi, H.; Bian, Y.; Guo, R. MiR-143 inhibits endometrial cancer cell proliferation and metastasis by targeting MAPK1. Oncotarget 2017, 8, 84384-84395. [CrossRef] [PubMed]

23. Chen, H.; Fan, Y.; Xu, W.; Chen, J.; Meng, Y.; Fang, D.; Wang, J. Exploration of miR-1202 and miR-196a in human endometrial cancer based on high throughout gene screening analysis. Oncol. Rep. 2017, 37, 3493-3501. [CrossRef] [PubMed]

24. Chen, H.X.; Xu, X.X.; Tan, B.Z.; Zhang, Z.; Zhou, X.D. MicroRNA-29b Inhibits Angiogenesis by Targeting VEGFA through the MAPK/ERK and PI3K/Akt Signaling Pathways in Endometrial Carcinoma. Cell. Physiol. Biochem. 2017, 41, 933-946. [CrossRef]

25. Chen, P.; Xing, T.; Wang, Q.; Liu, A.; Liu, H.; Hu, Y.; Ji, Y.; Song, Y.; Wang, D. MicroRNA-202 inhibits cell migration and invasion through targeting FGF2 and inactivating Wnt/ $\beta$-catenin signaling in endometrial carcinoma. Biosci. Rep. 2019, 39. [CrossRef]

26. Chen, R.; Zhang, M.; Liu, W.; Chen, H.; Cai, T.; Xiong, H.; Sheng, X.; Liu, S.; Peng, J.; Wang, F.; et al. Estrogen affects the negative feedback loop of PTENP1-miR200c to inhibit PTEN expression in the development of endometrioid endometrial carcinoma. Cell Death Dis. 2018, 10, 4. [CrossRef]

27. Chen, S.; Chen, X.; Sun, K.X.; Xiu, Y.L.; Liu, B.L.; Feng, M.X.; Sang, X.B.; Zhao, Y. MicroRNA-93 Promotes Epithelial-Mesenchymal Transition of Endometrial Carcinoma Cells. PLoS ONE 2016, 11, e0165776. [CrossRef]

28. Chen, Z.; Zhu, Y.; Fan, X.; Liu, Y.; Feng, Q. Decreased expression of miR-184 restrains the growth and invasion of endometrial carcinoma cells through CDC25A-dependent Notch signaling pathway. Am. J. Transl. Res. 2019, 11, 755-764.

29. Chung, T.K.; Cheung, T.H.; Huen, N.Y.; Wong, K.W.; Lo, K.W.; Yim, S.F.; Siu, N.S.; Wong, Y.M.; Tsang, P.T.; Pang, M.W.; et al. Dysregulated microRNAs and their predicted targets associated with endometrioid endometrial adenocarcinoma in Hong Kong women. Int. J. Cancer 2009, 124, 1358-1365. [CrossRef]

30. Chung, T.K.; Lau, T.S.; Cheung, T.H.; Yim, S.F.; Lo, K.W.; Siu, N.S.; Chan, L.K.; Yu, M.Y.; Kwong, J.; Doran, G.; et al. Dysregulation of microRNA-204 mediates migration and invasion of endometrial cancer by regulating FOXC1. Int. J. Cancer 2012, 130, 1036-1045. [CrossRef]

31. Dai, Y.; Xia, W.; Song, T.; Su, X.; Li, J.; Li, S.; Chen, Y.; Wang, W.; Ding, H.; Liu, X.; et al. MicroRNA-200b is overexpressed in endometrial adenocarcinomas and enhances MMP2 activity by downregulating TIMP2 in human endometrial cancer cell line HEC-1A cells. Nucleic Acid Ther. 2013, 23, 29-34. [CrossRef]

32. Dong, P. Mutant p53 gain-of-function induces epithelial-mesenchymal transition through modulation of the miR-130b-ZEB1 axis. J. Cancer Sci. Ther. 2012, 4, 131. [CrossRef]

33. Dong, P.; Ihira, K.; Xiong, Y.; Watari, H.; Hanley, S.J.; Yamada, T.; Hosaka, M.; Kudo, M.; Yue, J.; Sakuragi, N. Reactivation of epigenetically silenced miR-124 reverses the epithelial-to-mesenchymal transition and inhibits invasion in endometrial cancer cells via the direct repression of IQGAP1 expression. Oncotarget 2016, 7, 20260-20270. [CrossRef]

34. Dong, P.; Xiong, Y.; Yue, J.; Hanley, S.J.B.; Watari, H. miR-34a, miR-424 and miR-513 inhibit MMSET expression to repress endometrial cancer cell invasion and sphere formation. Oncotarget 2018, 9, 23253-23263. [CrossRef] 
35. Dong, Y.; Si, J.W.; Li, W.T.; Liang, L.; Zhao, J.; Zhou, M.; Li, D.; Li, T. miR-200a/miR-141 and miR-205 upregulation might be associated with hormone receptor status and prognosis in endometrial carcinomas. Int. J. Clin. Exp. Pathol. 2015, 8, $2864-2875$.

36. Fang, Y.Y.; Tan, M.R.; Zhou, J.; Liang, L.; Liu, X.Y.; Zhao, K.; Bao, E.C. MiR-214-3p inhibits epithelial-to-mesenchymal transition and metastasis of endometrial cancer cells by targeting TWISTI. OncoTargets Ther. 2019, 12, 9449-9458. [CrossRef]

37. He, S.; Zeng, S.; Zhou, Z.W.; He, Z.X.; Zhou, S.F. Hsa-microRNA-181a is a regulator of a number of cancer genes and a biomarker for endometrial carcinoma in patients: A bioinformatic and clinical study and the therapeutic implication. Drug Des. Dev. Ther. 2015, 9, 1103-1175. [CrossRef]

38. Hiroki, E.; Akahira, J.; Suzuki, F.; Nagase, S.; Ito, K.; Suzuki, T.; Sasano, H.; Yaegashi, N. Changes in microRNA expression levels correlate with clinicopathological features and prognoses in endometrial serous adenocarcinomas. Cancer Sci. 2010, 101, 241-249. [CrossRef]

39. Hiroki, E.; Suzuki, F.; Akahira, J.I.; Nagase, S.; Ito, K.; Sugawara, J.I.; Miki, Y.; Suzuki, T.; Sasano, H.; Yaegashi, N. MicroRNA-34b functions as a potential tumor suppressor in endometrial serous adenocarcinoma. Int. J. Cancer 2012, 131, E395-E404. [CrossRef]

40. Huang, Y.; Yang, N. MicroRNA-20a-5p inhibits epithelial to mesenchymal transition and invasion of endometrial cancer cells by targeting STAT3. Int. J. Clin. Exp. Pathol. 2018, 11, 5715-5724.

41. Ihira, K.; Dong, P.; Xiong, Y.; Watari, H.; Konno, Y.; Hanley, S.J.; Noguchi, M.; Hirata, N.; Suizu, F.; Yamada, T.; et al. EZH2 inhibition suppresses endometrial cancer progression via miR-361/Twist axis. Oncotarget 2017, 8, 13509-13520. [CrossRef]

42. Jiang, F.Z.; He, Y.Y.; Wang, H.H.; Zhang, H.L.; Zhang, J.; Yan, X.F.; Wang, X.J.; Che, Q.; Ke, J.Q.; Chen, Z.; et al. Mutant p53 induces EZH2 expression and promotes epithelial-mesenchymal transition by disrupting p68-Drosha complex assembly and attenuating miR-26a processing. Oncotarget 2015, 6, 44660-44674. [CrossRef]

43. Jiang, L.; Meng, W.; Zeng, J.; Hu, H.; Lu, L. MiR-34c oligonucleotide enhances chemosensitivity of Ishikawa cell to cisplatin by inducing apoptosis. Cell Biol. Int. 2013, 37, 577-583. [CrossRef]

44. Jiang, T.; Sui, D.; You, D.; Yao, S.; Zhang, L.; Wang, Y.; Zhao, J.; Zhang, Y. MiR-29a-5p inhibits proliferation and invasion and induces apoptosis in endometrial carcinoma via targeting TPX2. Cell Cycle 2018, 17, 1268-1278. [CrossRef]

45. Jing, L.; Hua, X.; Yuanna, D.; Rukun, Z.; Junjun, M. Exosomal miR-499a-5p Inhibits Endometrial Cancer Growth and Metastasis via Targeting VAV3. Cancer Manag. Res. 2020, 12, 13541-13552. [CrossRef]

46. Konno, Y.; Dong, P.; Xiong, Y.; Suzuki, F.; Lu, J.; Cai, M.; Watari, H.; Mitamura, T.; Hosaka, M.; Hanley, S.J.; et al. MicroRNA-101 targets EZH2, MCL-1 and FOS to suppress proliferation, invasion and stem cell-like phenotype of aggressive endometrial cancer cells. Oncotarget 2014, 5, 6049-6062. [CrossRef]

47. Kottaridi, C.; Spathis, A.; Margari, N.; Koureas, N.; Terzakis, E.; Chrelias, C.; Pappas, A.; Bilirakis, E.; Pouliakis, A.; Panayiotides, I.J.; et al. Evaluation Analysis of miRNAs Overexpression in Liquid-Based Cytology Endometrial Samples. J. Cancer 2017, 8 , 2699-2703. [CrossRef]

48. Lee, H.; Choi, H.J.; Kang, C.S.; Lee, H.J.; Lee, W.S.; Park, C.S. Expression of miRNAs and PTEN in endometrial specimens ranging from histologically normal to hyperplasia and endometrial adenocarcinoma. Mod. Pathol. Off. J. USA Acad. Pathol. Inc. 2012, 25, 1508-1515. [CrossRef] [PubMed]

49. Li, Y.; Sun, D.; Gao, J.; Shi, Z.; Chi, P.; Meng, Y.; Zou, C.; Wang, Y. MicroRNA-373 promotes the development of endometrial cancer by targeting LATS2 and activating the Wnt/ $\beta$-Catenin pathway. J. Cell. Biochem. 2018. [CrossRef] [PubMed]

50. Li, Y.; Zhang, Z.; Liu, X.; Huang, T.; He, W.; Shen, Y.; Liu, X.; Hong, K.; Cao, Q. miR-124 functions as a tumor suppressor in the endometrial carcinoma cell line HEC-1B partly by suppressing STAT3. Mol. Cell. Biochem. 2014, 388, 219-231. [CrossRef] [PubMed]

51. Li, Y.; Zhang, Z.; Zhang, X.; Lin, Y.; Luo, T.; Xiao, Z.; Zhou, Q. A dual PI3K/AKT/mTOR signaling inhibitor miR-99a suppresses endometrial carcinoma. Am. J. Transl. Res. 2016, 8, 719-731.

52. Liu, B.; Che, Q.; Qiu, H.; Bao, W.; Chen, X.; Lu, W.; Li, B.; Wan, X. Elevated MiR-222-3p promotes proliferation and invasion of endometrial carcinoma via targeting ER $\alpha$. PLoS ONE 2014, 9, e87563. [CrossRef]

53. Li, Q.; Zhang, C.; Chen, R.; Xiong, H.; Qiu, F.; Liu, S.; Zhang, M.; Wang, F.; Wang, Y.; Zhou, X.; et al. Disrupting MALAT1/miR-200c sponge decreases invasion and migration in endometrioid endometrial carcinoma. Cancer Lett. 2016, 383, 28-40. [CrossRef]

54. Liu, J.H.; Li, C.Y.; Jiang, Y.; Wan, Y.C.; Zhou, S.L.; Cheng, W.J. Tumor-Suppressor role of miR-139-5p in endometrial cancer. Cancer Cell Int. 2018, 18. [CrossRef]

55. Liu, W.; Zhang, B.; Xu, N.; Wang, M.J.; Liu, Q. miR-326 regulates EMT and metastasis of endometrial cancer through targeting TWIST1. Eur. Rev. Med. Pharmacol. Sci. 2017, 21, 3787-3793.

56. Liu, Y.; Li, H.; Zhao, C.; Jia, H. MicroRNA-101 inhibits angiogenesis via COX-2 in endometrial carcinoma. Mol. Cell. Biochem. 2018, 448, 61-69. [CrossRef]

57. Lu, L.; Shen, Y.; Tseng, K.; Jiang, L.; Liu, W.; Duan, H.; Meng, W. Oncogenic function of miR-301 to predicts poor prognosis of endometrial cancer. Int. J. Clin. Exp. Med. 2016, 9, 12937-12942.

58. Lu, Z.; Nian, Z.; Jingjing, Z.; Tao, L.; Quan, L. MicroRNA-424/E2F6 feedback loop modulates cell invasion, migration and EMT in endometrial carcinoma. Oncotarget 2017, 8, 114281-114291. [CrossRef]

59. Ma, Y.J.; Ha, C.F.; Bai, Z.M.; Li, H.N.; Xiong, Y.; Jiang, J. Overexpression of microRNA-205 predicts lymph node metastasis and indicates an unfavorable prognosis in endometrial cancer. Oncol. Lett. 2016, 12, 4403-4410. [CrossRef]

60. Mozos, A.; Catasús, L.; D’Angelo, E.; Serrano, E.; Espinosa, I.; Ferrer, I.; Pons, C.; Prat, J. The FOXO1-miR27 tandem regulates myometrial invasion in endometrioid endometrial adenocarcinoma. Hum. Pathol. 2014, 45, 942-951. [CrossRef] 
61. Notaro, S.; Reimer, D.; Duggan-Peer, M.; Fiegl, H.; Wiedermair, A.; Rössler, J.; Altevogt, P.; Marth, C.; Zeimet, A.G. Evaluating L1CAM expression in human endometrial cancer using qRT-PCR. Oncotarget 2016, 7, 40221-40232. [CrossRef]

62. Ruan, H.; Liang, X.; Zhao, W.; Ma, L.; Zhao, Y. The effects of microRNA-183 promots cell proliferation and invasion by targeting MMP-9 in endometrial cancer. Biomed. Pharmacother. Biomed. Pharmacother. 2017, 89, 812-818. [CrossRef]

63. Shang, C.; Lu, Y.M.; Meng, L.R. MicroRNA-125b down-regulation mediates endometrial cancer invasion by targeting ERBB2. Med. Sci. Monit. Int. Med. J. Exp. Clin. Res. 2012, 18, BR149-BR155. [CrossRef]

64. Shao, W.; Li, Y.; Chen, F.; Jia, H.; Jia, J.; Fu, Y. Long non-coding RNA DLEU1 contributes to the development of endometrial cancer by sponging miR-490 to regulate SP1 expression. Pharmazie 2018, 73, 379-385. [CrossRef]

65. Shi, Y.; Jia, L.; Wen, H. Circ_0109046 Promotes the Progression of Endometrial Cancer via Regulating miR-136/HMGA2 Axis. Cancer Manag. Res. 2020, 12, 10993-11003. [CrossRef]

66. Shu, S.; Liu, X.; Xu, M.; Gao, X.; Fan, J.; Liu, H.; Li, R. MicroRNA-424 regulates epithelial-mesenchymal transition of endometrial carcinoma by directly targeting insulin-like growth factor 1 receptor. J. Cell. Biochem. 2019, 120, 2171-2179. [CrossRef] [PubMed]

67. Song, N.; Zhang, Y.; Kong, F.; Yang, H.; Ma, X. HOXA-AS2 promotes type I endometrial carcinoma via miRNA-302c-3p-mediated regulation of ZFX. Cancer Cell Int. 2020, 20, 359. [CrossRef] [PubMed]

68. Su, N.; Qiu, H.; Chen, Y.; Yang, T.; Yan, Q.; Wan, X. miR-205 promotes tumor proliferation and invasion through targeting ESRRG in endometrial carcinoma. Oncol. Rep. 2013, 29, 2297-2302. [CrossRef] [PubMed]

69. Su, Y.; Wang, J.; Ma, Z.; Gong, W.; Yu, L. miR-142 Suppresses Endometrial Cancer Proliferation In Vitro and In Vivo by Targeting Cyclin D1. DNA Cell Biol. 2019, 38, 144-150. [CrossRef] [PubMed]

70. Sun, K.X.; Chen, Y.; Chen, S.; Liu, B.L.; Feng, M.X.; Zong, Z.H.; Zhao, Y. The correlation between microRNA490-3p and TGF $\alpha$ in endometrial carcinoma tumorigenesis and progression. Oncotarget 2016, 7, 9236-9249. [CrossRef]

71. Sun, X.; Cui, M.; Zhang, A.; Tong, L.; Wang, K.; Li, K.; Wang, X.; Sun, Z.; Zhang, H. MiR-548c impairs migration and invasion of endometrial and ovarian cancer cells via downregulation of Twist. J. Exp. Clin. Cancer Res. 2016, 35, 10. [CrossRef]

72. Sun, X.; Dongol, S.; Qiu, C.; Xu, Y.; Sun, C.; Zhang, Z.; Yang, X.; Zhang, Q.; Kong, B. miR-652 Promotes Tumor Proliferation and Metastasis by Targeting RORA in Endometrial Cancer. Mol. Cancer Res. 2018, 16, 1927-1939. [CrossRef]

73. Tang, W.; Li, J.; Liu, H.; Zhou, F.; Liu, M. MiR-106a promotes tumor growth, migration, and invasion by targeting BCL2L11 in human endometrial adenocarcinoma. Am. J. Transl. Res. 2017, 9, 4984-4993.

74. Tian, Y.; Chen, Y.Y.; Han, A.L. MiR-1271 inhibits cell proliferation and metastasis by targeting LDHA in endometrial cancer. Eur. Rev. Med. Pharmacol. Sci. 2019, 23, 5648-5656. [CrossRef]

75. Torres, A.; Torres, K.; Paszkowski, T.; Radej, S.; Staśkiewicz, G.J.; Ceccaroni, M.; Pesci, A.; Maciejewski, R. Highly increased maspin expression corresponds with up-regulation of miR-21 in endometrial cancer: A preliminary report. Int. J. Gynecol. Cancer Off. J. Int. Gynecol. Cancer Soc. 2011, 21, 8-14. [CrossRef]

76. Torres, A.; Torres, K.; Pesci, A.; Ceccaroni, M.; Paszkowski, T.; Cassandrini, P.; Zamboni, G.; Maciejewski, R. Deregulation of miR-100, miR-99a and miR-199b in tissues and plasma coexists with increased expression of mTOR kinase in endometrioid endometrial carcinoma. BMC Cancer 2012, 12. [CrossRef]

77. Torres, A.; Torres, K.; Pesci, A.; Ceccaroni, M.; Paszkowski, T.; Cassandrini, P.; Zamboni, G.; Maciejewski, R. Diagnostic and prognostic significance of miRNA signatures in tissues and plasma of endometrioid endometrial carcinoma patients. Int. J. Cancer 2013, 132, 1633-1645. [CrossRef]

78. Tsukamoto, O.; Miura, K.; Mishima, H.; Abe, S.; Kaneuchi, M.; Higashijima, A.; Miura, S.; Kinoshita, A.; Yoshiura, K.; Masuzaki, H. Identification of endometrioid endometrial carcinoma-associated microRNAs in tissue and plasma. Gynecol. Oncol. 2014, 132, 715-721. [CrossRef]

79. Tu, C.; Wang, F.; Wan, J. MicroRNA-381 inhibits cell proliferation and invasion in endometrial carcinoma by targeting the IGF-1R. Mol. Med. Rep. 2018, 17, 4090-4098. [CrossRef]

80. Wang, C.; Su, K.; Zhang, Y.; Zhang, W.; Chu, D.; Zhao, Q.; Guo, R. MicroRNA-365 targets multiple oncogenes to inhibit proliferation, invasion, and self-renewal of aggressive endometrial cancer cells. Cancer Manag. Res. 2018, 10, 5171-5185. [CrossRef]

81. Wang, H.; Wang, T.T.; Lv, X.P. Expression and prognostic value of miRNA-29b in peripheral blood for endometrial cancer. Future Oncol. 2018, 14, 1365-1376. [CrossRef]

82. Wang, J.; Zhao, X.; Guo, Z.; Ma, X.; Song, Y.; Guo, Y. Regulation of NEAT1/miR-214-3p on the growth, migration and invasion of endometrial carcinoma cells. Arch. Gynecol. Obstet. 2017, 295, 1469-1475. [CrossRef]

83. Wang, L.; Zhao, S.; Mingxin, Y.U. LncRNA NR2F1-AS1 is involved in the progression of endometrial cancer by sponging miR-363 to target SOX4. Pharmazie 2019, 74, 295-300. [CrossRef]

84. Wang, Q.; Zhu, W. MicroRNA-873 inhibits the proliferation and invasion of endometrial cancer cells by directly targeting hepatoma-derived growth factor. Exp. Ther. Med. 2019, 18, 1291-1298. [CrossRef]

85. Wang, W.; Ge, L.; Xu, X.J.; Yang, T.; Yuan, Y.; Ma, X.L.; Zhang, X.H. LncRNA NEAT1 promotes endometrial cancer cell proliferation, migration and invasion by regulating the miR-144-3p/EZH2 axis. Radiol. Oncol. 2019, 53, 434-442. [CrossRef]

86. Wang, Y.; Dong, L.; Liu, Y. Targeting Thyroid Receptor Interacting Protein 6 by MicroRNA-589-5p Inhibits Cell Proliferation, Migration, and Invasion in Endometrial Carcinoma. Cancer Biother. Radiopharm. 2019, 34, 529-536. [CrossRef]

87. Wang, Y.; Yin, L. LINC00461 Promoted Endometrial Carcinoma Growth and Migration by Targeting MicroRNA-2195p/Cyclooxygenase-2 Signaling Axis. Cell Transplant. 2021, 30. [CrossRef] 
88. Wei, D.; Tian, M.; Fan, W.; Zhong, X.; Wang, S.; Chen, Y.; Zhang, S. Circular RNA circ_0000043 promotes endometrial carcinoma progression by regulating miR-1271-5p/CTNND1 axis. Arch. Gynecol. Obstet. 2020. [CrossRef]

89. Wilczynski, M.; Danielska, J.; Dzieniecka, M.; Szymanska, B.; Wojciechowski, M.; Malinowski, A. Prognostic and Clinical Significance of miRNA-205 in Endometrioid Endometrial Cancer. PLoS ONE 2016, 11, e0164687. [CrossRef]

90. Wu, D.; Huang, H.J.; He, C.N.; Wang, K.Y. MicroRNA-199a-3p regulates endometrial cancer cell proliferation by targeting mammalian target of rapamycin (mTOR). Int. J. Gynecol. Cancer Off. J. Int. Gynecol. Cancer Soc. 2013, 23, 1191-1197. [CrossRef]

91. Xin, W.; Gao, X.; Zhao, S.; Zhao, P.; Yu, H.; Wu, Q.; Hua, K. LncRNA RP11-395G23.3 suppresses the endometrial cancer progression via regulating microRNA-205-5p/PTEN axis. Am. J. Transl. Res. 2020, 12, 4422-4433. [PubMed]

92. Xiong, H.; Chen, R.; Liu, S.; Lin, Q.; Chen, H.; Jiang, Q. MicroRNA-183 induces epithelial-mesenchymal transition and promotes endometrial cancer cell migration and invasion in by targeting CPEB1. J. Cell. Biochem. 2018, 119, 8123-8137. [CrossRef] [PubMed]

93. Xiong, H.; Wang, N.; Chen, H.; Zhang, M.; Lin, Q. MicroRNA-199a/b-5p inhibits endometrial cancer cell metastasis and invasion by targeting FAM83B in the epithelial-to-mesenchymal transition signaling pathway. Mol. Med. Rep. 2021, 23. [CrossRef] [PubMed]

94. Xu, C.; Zhai, J.; Fu, Y. Overexpression of Nuclear Enriched Autosomal Transcript 1 Facilitates Cell Proliferation, Migration Invasion, and Suppresses Apoptosis in Endometrial Cancer by Targeting MicroRNA-202-3p/T Cell Immunoglobulin and Mucin Domain 4 Axis. Cancer Biother. Radiopharm. 2020. [CrossRef]

95. Xu, J.B. MicroRNA-93-5p/IFNAR1 axis accelerates metastasis of endometrial carcinoma by activating the STAT3 pathway. Eur. Rev. Med. Pharmacol. Sci. 2019, 23, 5657-5666. [CrossRef]

96. Xu, X.; Kong, X.; Liu, T.; Zhou, L.; Wu, J.; Fu, J.; Wang, Y.; Zhu, M.; Yao, S.; Ding, Y.; et al. Metastasis-associated protein 1, modulated by miR-30c, promotes endometrial cancer progression through AKT/mTOR/4E-BP1 pathway. Gynecol. Oncol. 2019, 154, 207-217. [CrossRef]

97. Yamamoto, N.; Nishikawa, R.; Chiyomaru, T.; Goto, Y.; Fukumoto, I.; Usui, H.; Mitsuhashi, A.; Enokida, H.; Nakagawa, M.; Shozu, M.; et al. The tumor-suppressive microRNA-1/133a cluster targets PDE7A and inhibits cancer cell migration and invasion in endometrial cancer. Int. J. Oncol. 2015, 47, 325-334. [CrossRef]

98. Yan, H.; Sun, B.M.; Zhang, Y.Y.; Li, Y.J.; Huang, C.X.; Feng, F.Z.; Li, C. Upregulation of miR-183-5p is responsible for the promotion of apoptosis and inhibition of the epithelial-mesenchymal transition, proliferation, invasion and migration of human endometrial cancer cells by downregulating Ezrin. Int. J. Mol. Med. 2018, 42, 2469-2480. [CrossRef]

99. Yang, B.; Li, W.; Ma, X.; Guo, Y.; Shen, G. The correlation between tumor tissue mir-373 and mir-124 expressions and prognosis in patients with endometrial cancer. Int. J. Clin. Exp. Med. 2020, 13, 4470-4477.

100. Yang, L.; Yang, Z.; Yao, R.; Li, Y.; Liu, Z.; Chen, X.; Zhang, G. miR-210 promotes progression of endometrial carcinoma by regulating the expression of NFIX. Int. J. Clin. Exp. Pathol. 2018, 11, 5213-5222.

101. Yanokura, M.; Banno, K.; Aoki, D. MicroRNA-34b expression enhances chemosensitivity of endometrial cancer cells to paclitaxel. Int. J. Oncol. 2020, 57, 1145-1156. [CrossRef]

102. Yoneyama, K.; Ishibashi, O.; Kawase, R.; Kurose, K.; Takeshita, T. miR-200a, miR-200b and miR-429 are onco-miRs that target the PTEN gene in endometrioid endometrial carcinoma. Anticancer Res. 2015, 35, 1401-1410.

103. Zhang, H.H.; Li, R.; Li, Y.J.; Yu, X.X.; Sun, Q.N.; Li, A.Y.; Kong, Y. eIF4E-related miR-320a and miR-340-5p inhibit endometrial carcinoma cell metastatic capability by preventing TGF- $\beta 1$-induced epithelial-mesenchymal transition. Oncol. Rep. 2020, 43, 447-460. [CrossRef]

104. Zhang, H.M.; Fan, T.T.; Li, W.; Li, X.X. Expressions and significances of TTF-1 and PTEN in early endometrial cancer. Eur. Rev. Med. Pharmacol. Sci. 2017, 21, 20-26.

105. Zhang, K.; Cai, Y.; Zhou, Q.; Sun, H.; Wei, J. Long non-coding RNA SNHG14 impedes viability, migration and invasion of endometrial carcinoma cells through modulating miR-93-5p/ ZBTB7A axis. Cancer Manag. Res. 2020, 12, 9515-9525. [CrossRef]

106. Zhang, S.; Wang, M.; Li, Q.; Zhu, P. MiR-101 reduces cell proliferation and invasion and enhances apoptosis in endometrial cancer via regulating PI3K/Akt/mTOR. Cancer Biomark. Sect. A Dis. Markers 2017, 21, 179-186. [CrossRef]

107. Zhao, W.; Geng, P.; Li, Y.; Wei, X.; Cheng, J. MicroRNA-21 promotes endometrial carcinoma proliferation and invasion by targeting PTEN. Int. J. Clin. Exp. Pathol. 2017, 10, 11489-11495.

108. Zhao, X.; Dai, L.; Yue, Q.; Wang, H.; Wang, X.U.; Li, Y.; Chen, R. MiR-195 inhibits migration, invasion and epithelial-mesenchymal transition (EMT) of endometrial carcinoma cells by targeting SOX4. J. Biosci. 2019, 44, 146. [CrossRef]

109. Zhao, X.; Zhu, D.; Lu, C.; Yan, D.; Li, L.; Chen, Z. MicroRNA-126 inhibits the migration and invasion of endometrial cancer cells by targeting insulin receptor substrate 1. Oncol. Lett. 2016, 11, 1207-1212. [CrossRef]

110. Zheng, W.; Yang, J.; Wang, Y.; Liu, X. Exosomal miRNA-93 and miRNA-205 expression in endometrial cancer. J. King Saud Univ. Sci. 2020, 32, 1111-1115. [CrossRef]

111. Zheng, W.P.; Meng, F.L.; Wang, L.Y. miR-544a Stimulates endometrial carcinoma growth via targeted inhibition of reversioninducing cysteine-rich protein with Kazal motifs. Mol. Cell. Probes 2020, 53, 101572. [CrossRef] [PubMed]

112. Zheng, X.; Xu, K.; Zhu, L.; Mao, M.; Zhang, F.; Cui, L. MiR-486-5p Act as a Biomarker in Endometrial Carcinoma: Promotes Cell Proliferation, Migration, Invasion by Targeting MARK1. OncoTargets Ther. 2020, 13, 4843-4853. [CrossRef] [PubMed]

113. Zhou, Y.X.; Wang, C.; Mao, L.W.; Wang, Y.L.; Xia, L.Q.; Zhao, W.; Shen, J.; Chen, J. Long noncoding RNA HOTAIR mediates the estrogen-induced metastasis of endometrial cancer cells via the miR-646/NPM1 axis. Am. J. Physiol. Cell Physiol. 2018, 314, C690-C701. [CrossRef] [PubMed] 
114. Zhou, Y.X.; Zhao, W.; Mao, L.W.; Wang, Y.L.; Xia, L.Q.; Cao, M.; Shen, J.; Chen, J. Long non-coding RNA NIFK-AS1 inhibits M2 polarization of macrophages in endometrial cancer through targeting miR-146a. Int. J. Biochem. Cell Biol. 2018, 104, 25-33. [CrossRef]

115. Zhou, Z.; Xu, Y.P.; Wang, L.J.; Kong, Y. miR-940 potentially promotes proliferation and metastasis of endometrial carcinoma through regulation of MRVI1. Biosci. Rep. 2019, 39. [CrossRef]

116. Zhu, H.; Jin, Y.M.; Lyu, X.M.; Fan, L.M.; Wu, F. Long noncoding RNA H19 regulates HIF-1 $\alpha /$ AXL signaling through inhibiting miR-20b-5p in endometrial cancer. Cell Cycle 2019, 18, 2454-2464. [CrossRef]

117. Zhu, L.; Wang, X.; Wang, T.; Zhu, W.; Zhou, X. MiR-494-3p promotes the progression of endometrial cancer by regulating the PTEN/PI3K/AKT pathway. Mol. Med. Rep. 2019, 19, 581-588. [CrossRef]

118. Ritter, A.; Hirschfeld, M.; Berner, K.; Jaeger, M.; Grundner-Culemann, F.; Schlosser, P.; Asberger, J.; Weiss, D.; Noethling, C.; Mayer, S.; et al. Discovery of potential serum and urine-based microRNA as minimally-invasive biomarkers for breast and gynecological cancer. Cancer Biomark. Sect. A Dis. Markers 2020, 27, 225-242. [CrossRef]

119. Wang, Y.; Zhang, S. Berberine suppresses growth and metastasis of endometrial cancer cells via miR-101/COX-2. Biomed. Pharmacother. Biomed. Pharmacother. 2018, 103, 1287-1293. [CrossRef]

120. Zheng, X.; Liu, M.; Song, Y.; Feng, C. Long Noncoding RNA-ATB Impairs the Function of Tumor Suppressor miR-126-Mediated Signals in Endometrial Cancer for Tumor Growth and Metastasis. Cancer Biother. Radiopharm. 2019, 34, 47-55. [CrossRef]

121. Li, S.; Hu, R.; Wang, C.; Guo, F.; Li, X.; Wang, S. miR-22 inhibits proliferation and invasion in estrogen receptor $\alpha$-positive endometrial endometrioid carcinomas cells. Mol. Med. Rep. 2014, 9, 2393-2399. [CrossRef]

122. Li, B.L.; Lu, C.; Lu, W.; Yang, T.T.; Qu, J.; Hong, X.; Wan, X.P. MiR-130b is an EMT-related microRNA that targets DICER1 for aggression in endometrial cancer. Med. Oncol. 2013, 30. [CrossRef]

123. Li, F.; Chen, H.; Huang, Y.; Zhang, Q.; Xue, J.; Liu, Z.; Zheng, F. MiR-34c plays a role of tumor suppressor in HEC-1-B cells by targeting E2F3 protein. Oncol. Rep. 2015, 33, 3069-3074. [CrossRef]

124. Zheng, Y.; Yang, X.; Wang, C.; Zhang, S.; Wang, Z.; Li, M.; Wang, Y.; Wang, X.; Yang, X. HDAC6, modulated by miR-206, promotes endometrial cancer progression through the PTEN/AKT/mTOR pathway. Sci. Rep. 2020, 10, 3576. [CrossRef]

125. Li, X.C.; Hai, J.J.; Tan, Y.J.; Yue, Q.F.; Liu, L.J. MiR-218 suppresses metastasis and invasion of endometrial cancer via negatively regulating ADD2. Eur. Rev. Med. Pharmacol. Sci. 2019, 23, 1408-1417. [CrossRef]

126. Ma, J.; Li, D.; Kong, F.F.; Yang, D.; Yang, H.; Ma, X.X. miR-302a-5p/367-3p-HMGA2 axis regulates malignant processes during endometrial cancer development. J. Exp. Clin. Cancer Res. 2018, 37, 19. [CrossRef]

127. Ye, W.; Xue, J.; Zhang, Q.; Li, F.; Zhang, W.; Chen, H.; Huang, Y.; Zheng, F. MiR-449a functions as a tumor suppressor in endometrial cancer by targeting CDC25A. Oncol. Rep. 2014, 32, 1193-1199. [CrossRef]

128. Li, H.L.; Sun, J.J.; Ma, H.; Liu, S.J.; Li, N.; Guo, S.J.; Shi, Y.; Xu, Y.Y.; Qi, Z.Y.; Wang, Y.Q.; et al. MicroRNA-23a inhibits endometrial cancer cell development by targeting SIX1. Oncol. Lett. 2019, 18, 3792-3802. [CrossRef]

129. Chen, H.; Fan, Y.; Xu, W.; Chen, J.; Xu, C.; Wei, X.; Fang, D.; Feng, Y. miR-10b Inhibits Apoptosis and Promotes Proliferation and Invasion of Endometrial Cancer Cells via Targeting HOXB3. Cancer Biother. Radiopharm. 2016, 31, 225-231. [CrossRef]

130. Liu, B.L.; Sun, K.X.; Zong, Z.H.; Chen, S.; Zhao, Y. MicroRNA-372 inhibits endometrial carcinoma development by targeting the expression of the Ras homolog gene family member C (RhoC). Oncotarget 2016, 7, 6649-6664. [CrossRef]

131. Chen, S.; Sun, K.X.; Liu, B.L.; Zong, Z.H.; Zhao, Y. MicroRNA-505 functions as a tumor suppressor in endometrial cancer by targeting TGF- $\alpha$. Mol. Cancer 2016, 15, 11. [CrossRef]

132. Zhang, H.C.; Han, Y.Y.; Zhang, X.M.; Xiao, N.; Jiang, T.; Zhu, S.; Wang, E.P.; Chen, C.B. miR-522 facilitates the prosperities of endometrial carcinoma cells by directly binding to monoamine oxidase B. Kaohsiung J. Med. Sci. 2019, 35, 598-606. [CrossRef]

133. Bai, J.X.; Yan, B.; Zhao, Z.N.; Xiao, X.; Qin, W.W.; Zhang, R.; Jia, L.T.; Meng, Y.L.; Jin, B.Q.; Fan, D.M.; et al. Tamoxifen represses miR-200 microRNAs and promotes epithelial-to-mesenchymal transition by up-regulating c-Myc in endometrial carcinoma cell lines. Endocrinology 2013, 154, 635-645. [CrossRef]

134. Cai, P.; Wu, M.; Zhang, B.; Wu, S.; Wei, H.; Wei, L. Long non-coding RNA SNHG12 regulates cell proliferation, invasion and migration in endometrial cancer by targeting miR-4429. Mol. Med. Rep. 2020, 22, 2842-2850. [CrossRef]

135. Che, X.; Jian, F.; Chen, C.; Liu, C.; Liu, G.; Feng, W. PCOS serum-derived exosomal miR-27a-5p stimulates endometrial cancer cells migration and invasion. J. Mol. Endocrinol. 2020, 64, 1-12. [CrossRef]

136. Chen, S.; Sun, K.X.; Liu, B.L.; Zong, Z.H.; Zhao, Y. The role of glycogen synthase kinase-3 $\beta$ (GSK-3 $\beta$ ) in endometrial carcinoma: A carcinogenesis, progression, prognosis, and target therapy marker. Oncotarget 2016, 7, 27538-27551. [CrossRef]

137. Chen, S.; Zong, Z.H.; Wu, D.D.; Sun, K.X.; Liu, B.L.; Zhao, Y. The role of metastasis-associated in colon cancer 1 (MACC1) in endometrial carcinoma tumorigenesis and progression. Mol. Carcinog. 2017, 56, 1361-1371. [CrossRef] [PubMed]

138. Cochrane, D.R.; Spoelstra, N.S.; Howe, E.N.; Nordeen, S.K.; Richer, J.K. MicroRNA-200c mitigates invasiveness and restores sensitivity to microtubule-targeting chemotherapeutic agents. Mol. Cancer Ther. 2009, 8, 1055-1066. [CrossRef] [PubMed]

139. Deng, J.; Wang, W.; Yu, G.; Ma, X. MicroRNA-195 inhibits epithelial-mesenchymal transition by targeting G protein-coupled estrogen receptor 1 in endometrial carcinoma. Mol. Med. Rep. 2019, 20, 4023-4032. [CrossRef] [PubMed]

140. Dong, M.K.N.S.P. Micro rna-106b modulates epithelial-mesenchymal transition by targeting twist1 in invasive endometrial cancer cell lines. Int. J. Gynecol. Cancer 2013, 23, 1076.

141. Dong, P.; Kaneuchi, M.; Watari, H.; Hamada, J.; Sudo, S.; Ju, J.; Sakuragi, N. MicroRNA-194 inhibits epithelial to mesenchymal transition of endometrial cancer cells by targeting oncogene BMI-1. Mol. Cancer 2011, 10, 99. [CrossRef] 
142. Fang, Q.; Sang, L.; Du, S. Long noncoding RNA LINC00261 regulates endometrial carcinoma progression by modulating miRNA/FOXO1 expression. Cell Biochem. Funct. 2018, 36, 323-330. [CrossRef]

143. Gao, X.; Yu, L.; Zhang, J.; Xue, P. Silencing of long non-coding RNA LINC01106 suppresses the proliferation, migration and invasion of endometrial cancer cells through regulating the miR-449a/MET axis. OncoTargets Ther. 2020, 13, 9643-9655. [CrossRef]

144. Gao, Y.; Qian, H.; Tang, X.; Du, X.; Wang, G.; Zhang, H.; Ye, F.; Liu, T. Superparamagnetic iron oxide nanoparticle-mediated expression of miR-326 inhibits human endometrial carcinoma stem cell growth. Int. J. Nanomed. 2019, 14, 2719-2731. [CrossRef]

145. Gong, B.; Yue, Y.; Wang, R.; Zhang, Y.; Jin, Q.; Zhou, X. Overexpression of microRNA-194 suppresses the epithelial-mesenchymal transition in targeting stem cell transcription factor Sox3 in endometrial carcinoma stem cells. Tumour Biol. J. Int. Soc. Oncodev. Biol. Med. 2017, 39. [CrossRef]

146. Howe, E.N.; Cochrane, D.R.; Richer, J.K. Targets of miR-200c mediate suppression of cell motility and anoikis resistance. Breast Cancer Res. 2011, 13, R45. [CrossRef]

147. Hu, Y.; Wu, A.Y.; Xu, C.; Song, K.Q.; Wang, W.J.; Yin, X.; Di, W.; Hong, Z.B.; Qiu, L.H. MicroRNA-449a Inhibits Tumor Metastasis through AKT/ERK1/2 Inactivation by Targeting Steroid Receptor Coactivator (SRC) in Endometrial Cancer. J. Cancer 2019, 10, 547-555. [CrossRef]

148. Jia, J.; Guo, S.; Zhang, D.; Tian, X.; Xie, X. Exosomal-lncRNA DLEU1 Accelerates the Proliferation, Migration, and Invasion of Endometrial Carcinoma Cells by Regulating microRNA-E2F3. OncoTargets Ther. 2020, 13, 8651-8663. [CrossRef]

149. Jin, C.; Liang, R. miR-205 promotes epithelial-mesenchymal transition by targeting AKT signaling in endometrial cancer cells. $J$. Obstet. Gynaecol. Res. 2015, 41, 1653-1660. [CrossRef]

150. Kong, J.; He, X.; Wang, Y.; Li, J. Effect of microRNA-29b on proliferation, migration, and invasion of endometrial cancer cells. J. Int. Med. Res. 2019, 47, 3803-3817. [CrossRef]

151. Li, B.L.; Lu, W.; Qu, J.J.; Ye, L.; Du, G.Q.; Wan, X.P. Loss of exosomal miR-148b from cancer-associated fibroblasts promotes endometrial cancer cell invasion and cancer metastasis. J. Cell. Physiol. 2019, 234, 2943-2953. [CrossRef]

152. Li, F.; Liang, A.; Lv, Y.; Liu, G.; Jiang, A.; Liu, P. MicroRNA-200c Inhibits Epithelial-Mesenchymal Transition by Targeting the BMI-1 Gene Through the Phospho-AKT Pathway in Endometrial Carcinoma Cells In Vitro. Med. Sci. Monit. Int. Med. J. Exp. Clin. Res. 2017, 23, 5139-5149. [CrossRef]

153. Li, J.; Sun, H.; Liu, T.; Kong, J. MicroRNA-423 promotes proliferation, migration and invasion and induces chemoresistance of endometrial cancer cells. Exp. Ther. Med. 2018, 16, 4213-4224. [CrossRef]

154. Li, L.; Shou, H.; Wang, Q.; Liu, S. Investigation of the potential theranostic role of KDM5B/miR-29c signaling axis in paclitaxel resistant endometrial carcinoma. Gene 2019, 694, 76-82. [CrossRef]

155. Liu, Y.; Chen, S.; Zong, Z.H.; Guan, X.; Zhao, Y. CircRNA WHSC1 targets the miR-646/NPM1 pathway to promote the development of endometrial cancer. J. Cell. Mol. Med. 2020, 24, 6898-6907. [CrossRef]

156. Pandey, D.P.; Picard, D. miR-22 inhibits estrogen signaling by directly targeting the estrogen receptor alpha mRNA. Mol. Cell Biol. 2009, 29, 3783-3790. [CrossRef]

157. Shen, A.; Tong, X.; Li, H.; Chu, L.; Jin, X.; Ma, H.; Ouyang, Y. TPPP3 inhibits the proliferation, invasion and migration of endometrial carcinoma targeted with miR-1827. Clin. Exp. Pharmacol. Physiol. 2021. [CrossRef]

158. Shi, W.; Wang, X.L.; Ruan, L.; Fu, J.; Liu, F.; Qu, J. MiR-200a promotes epithelial-mesenchymal transition of endometrial cancer cells by negatively regulating FOXA2 expression. Pharmazie 2017, 72, 694-699. [CrossRef] [PubMed]

159. Wang, J.; Zhang, L.; Jiang, W.; Zhang, R.; Zhang, B.; Silayiding, A.; Duan, X. MicroRNA-135a promotes proliferation, migration, invasion and induces chemoresistance of endometrial cancer cells. Eur. J. Obstet. Gynecol. Reprod. Biol. X 2020, $5,100103$. [CrossRef] [PubMed]

160. Wu, A.Y.; Hu, Y.; Cang, W.; Li, D.; Wang, W.J.; Tian, Q.; Gu, L.Y.; Zhang, N.; Ji, F.; Qiu, L.H. Suppressive effect of microRNA-449a on the NDRG1/PTEN/AKT axis regulates endometrial cancer growth and metastasis. Exp. Cell Res. 2019, 382, 111468. [CrossRef] [PubMed]

161. Xia, X.; Wang, J.; Liu, Y.; Yue, M. Lower cystic fibrosis transmembrane conductance regulator (CFTR) promotes the proliferation and migration of endometrial carcinoma. Med. Sci. Monit. 2017, 23, 966-974. [CrossRef]

162. Xu, D.; Dong, P.; Xiong, Y.; Chen, R.; Konno, Y.; Ihira, K.; Yue, J.; Watari, H. PD-L1 Is a Tumor Suppressor in Aggressive Endometrial Cancer Cells and Its Expression Is Regulated by miR-216a and lncRNA MEG3. Front. Cell Dev. Biol. 2020, 8 , 598205. [CrossRef]

163. Yao, H.; Kong, F.; Zhou, Y. MiR-182 promotes cell proliferation, migration and invasion by targeting FoxF2 in endometrial carcinoma cells. Int. J. Clin. Exp. Pathol. 2019, 12, 1248-1259.

164. Yu, D.; Zhou, H.; Xun, Q.; Xu, X.; Ling, J.; Hu, Y. microRNA-103 regulates the growth and invasion of endometrial cancer cells through the downregulation of tissue inhibitor of metalloproteinase 3. Oncol. Lett. 2012, 3, 1221-1226. [CrossRef]

165. Zhong, Y.; Wang, Y.; Dang, H.; Wu, X. LncRNA AFAP1-AS1 contributes to the progression of endometrial carcinoma by regulating miR-545-3p/VEGFA pathway. Mol. Cell. Probes 2020, 53. [CrossRef]

166. Wang, Z.; Wang, W.; Huang, K.; Wang, Y.; Li, J.; Yang, X. MicroRNA-34a inhibits cells proliferation and invasion by downregulating Notch1 in endometrial cancer. Oncotarget 2017, 8, 111258-111270. [CrossRef]

167. Liu, S.; Qiu, J.; Tang, X.; Cui, H.; Zhang, Q.; Yang, Q. LncRNA-H19 regulates cell proliferation and invasion of ectopic endometrium by targeting ITGB3 via modulating miR-124-3p. Exp. Cell Res. 2019, 381, 215-222. [CrossRef] 
168. Chen, X.; Yan, Q.; Li, S.; Zhou, L.; Yang, H.; Yang, Y.; Liu, X.; Wan, X. Expression of the tumor suppressor miR-206 is associated with cellular proliferative inhibition and impairs invasion in ER $\alpha$-positive endometrioid adenocarcinoma. Cancer Lett. 2012, 314, 41-53. [CrossRef]

169. Dong, P.; Xiong, Y.; Yue, J.; Xu, D.; Ihira, K.; Konno, Y.; Kobayashi, N.; Todo, Y.; Watari, H. Long noncoding RNA NEAT1 drives aggressive endometrial cancer progression via miR-361-regulated networks involving STAT3 and tumor microenvironmentrelated genes. J. Exp. Clin. Cancer Res. 2019, 38, 295. [CrossRef]

170. Schirmer, U.; Doberstein, K.; Rupp, A.K.; Bretz, N.P.; Wuttig, D.; Kiefel, H.; Breunig, C.; Fiegl, H.; Müller-Holzner, E.; Zeillinger, R.; et al. Role of miR-34a as a suppressor of L1CAM in endometrial carcinoma. Oncotarget 2014, 5, 462-472. [CrossRef]

171. Asanoma, K.; Hori, E.; Yoshida, S.; Yagi, H.; Onoyama, I.; Kodama, K.; Yasunaga, M.; Ohgami, T.; Kaneki, E.; Okugawa, K.; et al. Mutual suppression between BHLHE40/BHLHE41 and the MIR301B-MIR130B cluster is involved in epithelial-to-mesenchymal transition of endometrial cancer cells. Oncotarget 2019, 10, 4640-4654. [CrossRef]

172. Qiu, L. MiR-449a suppresses endometrial cancer invasion and metastasis by targeting NDRG1. Ann. Oncol. 2018, 29 , viii11. [CrossRef]

173. Zhou, H.; Xu, X.; Xun, Q.; Yu, D.; Ling, J.; Guo, F.; Yan, Y.; Shi, J.; Hu, Y. microRNA-30c negatively regulates endometrial cancer cells by targeting metastasis-associated gene-1. Oncol. Rep. 2012, 27, 807-812. [CrossRef]

174. Van Nyen, T.; Moiola, C.P.; Colas, E.; Annibali, D.; Amant, F. Modeling Endometrial Cancer: Past, Present, and Future. Int. J. Mol. Sci. 2018, 19, 2348. [CrossRef]

175. Buchynska, L.G.; Borykun, T.V.; Iurchenko, N.P.; Nespryadko, S.V.; Nesina, I.P. Expression of microRNA in tumor cells of endmetrioid carcinoma of endometrium. Exp. Oncol. 2020, 42, 289-294. [CrossRef]

176. Canlorbe, G.; Wang, Z.; Laas, E.; Bendifallah, S.; Castela, M.; Lefevre, M.; Chabbert-Buffet, N.; Daraï, E.; Aractingi, S.; Méhats, C.; et al. Identification of microRNA expression profile related to lymph node status in women with early-stage grade 1-2 endometrial cancer. Mod. Pathol. Off. J. US Can. Acad. Pathol. Inc. 2016, 29, 391-401. [CrossRef]

177. Cohn, D.E.; Fabbri, M.; Valeri, N.; Alder, H.; Ivanov, I.; Liu, C.G.; Croce, C.M.; Resnick, K.E. Comprehensive miRNA profiling of surgically staged endometrial cancer. Am. J. Obstet. Gynecol. 2010, 202, 656.e1-656.e8. [CrossRef]

178. Lee, L.J.; Ratner, E.; Uduman, M.; Winter, K.; Boeke, M.; Greven, K.M.; King, S.; Burke, T.W.; Underhill, K.; Kim, H.; et al. The KRAS-variant and miRNA expression in RTOG endometrial cancer clinical trials 9708 and 9905. PLoS ONE 2014, 9, e94167. [CrossRef]

179. Xu, X.; Liu, T.; Wang, Y.; Fu, J.; Yang, Q.; Wu, J.; Zhou, H. MiRNA-mRNA associated with survival in endometrial cancer. Front. Genet. 2019, 10. [CrossRef]

180. Zhai, H.; Karaayvaz, M.; Dong, P.; Sakuragi, N.; Ju, J. Prognostic significance of miR-194 in endometrial cancer. Biomark. Res. 2013, 1, 12. [CrossRef] [PubMed]

181. Romero-Pérez, L.; López-García, M.Á.; Díaz-Martín, J.; Biscuola, M.; Castilla, M.Á.; Tafe, L.J.; Garg, K.; Oliva, E.; Matias-Guiu, X.; Soslow, R.A.; et al. ZEB1 overexpression associated with E-cadherin and microRNA-200 downregulation is characteristic of undifferentiated endometrial carcinoma. Mod. Pathol. 2013, 26, 1514-1524. [CrossRef] [PubMed]

182. Ravo, M.; Cordella, A.; Rinaldi, A.; Bruno, G.; Alexandrova, E.; Saggese, P.; Nassa, G.; Giurato, G.; Tarallo, R.; Marchese, G.; et al. Small non-coding RNA deregulation in endometrial carcinogenesis. Oncotarget 2015, 6, 4677-4691. [CrossRef] [PubMed]

183. Donkers, H.; Bekkers, R.; Galaal, K. Diagnostic value of microRNA panel in endometrial cancer: A systematic review. Oncotarget 2020, 11, 2010-2023. [CrossRef] [PubMed]

184. Delangle, R.; De Foucher, T.; Larsen, A.K.; Sabbah, M.; Azaïs, H.; Bendifallah, S.; Daraï, E.; Ballester, M.; Mehats, C.; Uzan, C.; et al. The Use of microRNAs in the Management of Endometrial Cancer: A Meta-Analysis. Cancers 2019, 11, 832. [CrossRef]

185. Favier, A.; Rocher, G.; Larsen, A.K.; Delangle, R.; Uzan, C.; Sabbah, M.; Castela, M.; Duval, A.; Mehats, C.; Canlorbe, G. MicroRNA as Epigenetic Modifiers in Endometrial Cancer: A Systematic Review. Cancers 2021, 13, 1137. [CrossRef]

186. Ouyang, T.; Liu, Z.; Han, Z.; Ge, Q. MicroRNA Detection Specificity: Recent Advances and Future Perspective. Anal. Chem. 2019, 91, 3179-3186. [CrossRef]

187. Kent, C.N.; Guttilla Reed, I.K. Regulation of epithelial-mesenchymal transition in endometrial cancer: Connecting PI3K, estrogen signaling, and microRNAs. Clin. Transl. Oncol. 2016, 18, 1056-1061. [CrossRef]

188. Chiu, H.C.; Li, C.J.; Yiang, G.T.; Tsai, A.P.; Wu, M.Y. Epithelial to Mesenchymal Transition and Cell Biology of Molecular Regulation in Endometrial Carcinogenesis. J. Clin. Med. 2019, 8, 439. [CrossRef]

189. Ribatti, D.; Tamma, R.; Annese, T. Epithelial-Mesenchymal Transition in Cancer: A Historical Overview. Transl. Oncol. 2020, 13, 100773. [CrossRef]

190. Tang, H.; Massi, D.; Hemmings, B.A.; Mandalà, M.; Hu, Z.; Wicki, A.; Xue, G. AKT-ions with a TWIST between EMT and MET. Oncotarget 2016, 7, 62767-62777. [CrossRef]

191. Colas, E.; Pedrola, N.; Devis, L.; Ertekin, T.; Campoy, I.; Martínez, E.; Llauradó, M.; Rigau, M.; Olivan, M.; Garcia, M.; et al. The EMT signaling pathways in endometrial carcinoma. Clin. Transl. Oncol. Off. Publ. Fed. Span. Oncol. Soc. Natl. Cancer Inst. Mex. 2012, 14, 715-720. [CrossRef]

192. Leal-Esteban, L.C.; Fajas, L. Cell cycle regulators in cancer cell metabolism. Biochim. Biophys. Acta. Mol. Basis Dis. 2020, 1866, 165715. [CrossRef]

193. Ueno, D.; Dancik, G.M.; Shuch, B. The Cell Cycle Progression Score: Unclear Role in Renal Cell Carcinoma. Eur. Urol. 2018, 74, 128-129. [CrossRef] 
194. Giannone, G.; Tuninetti, V.; Ghisoni, E.; Genta, S.; Scotto, G.; Mittica, G.; Valabrega, G. Role of Cyclin-Dependent Kinase Inhibitors in Endometrial Cancer. Int. J. Mol. Sci. 2019, 20, 2353. [CrossRef]

195. Witek, Ł.; Janikowski, T.; Gabriel, I.; Bodzek, P.; Olejek, A. Analysis of microRNA regulating cell cycle-related tumor suppressor genes in endometrial cancer patients. Hum. Cell 2021, 34, 564-569. [CrossRef]

196. Xu, J.; Lin, D.I. Oncogenic c-terminal cyclin D1 (CCND1) mutations are enriched in endometrioid endometrial adenocarcinomas PLoS ONE 2018, 13, e0199688. [CrossRef]

197. Brooks, R.A.; Fleming, G.F.; Lastra, R.R.; Lee, N.K.; Moroney, J.W.; Son, C.H.; Tatebe, K.; Veneris, J.L. Current recommendations and recent progress in endometrial cancer. CA Cancer J. Clin. 2019, 69, 258-279. [CrossRef]

198. Mitsuhashi, Y.; Horiuchi, A.; Miyamoto, T.; Kashima, H.; Suzuki, A.; Shiozawa, T. Prognostic significance of Notch signalling molecules and their involvement in the invasiveness of endometrial carcinoma cells. Histopathology 2012, 60, 826-837. [CrossRef]

199. Wang, Z.; Li, Y.; Kong, D.; Banerjee, S.; Ahmad, A.; Azmi, A.S.; Ali, S.; Abbruzzese, J.L.; Gallick, G.E.; Sarkar, F.H. Acquisition of epithelial-mesenchymal transition phenotype of gemcitabine-resistant pancreatic cancer cells is linked with activation of the notch signaling pathway. Cancer Res. 2009, 69, 2400-2407. [CrossRef]

200. Mayor, R.; Etienne-Manneville, S. The front and rear of collective cell migration. Nat. Rev. Mol. Cell Biol. 2016, 17, 97-109. [CrossRef]

201. Ong, M.S.; Deng, S.; Halim, C.E.; Cai, W.; Tan, T.Z.; Huang, R.Y.-J.; Sethi, G.; Hooi, S.C.; Kumar, A.P.; Yap, C.T. Cytoskeletal Proteins in Cancer and Intracellular Stress: A Therapeutic Perspective. Cancers 2020, 12, 238. [CrossRef]

202. Köbel, M.; Langhammer, T.; Hüttelmaier, S.; Schmitt, W.D.; Kriese, K.; Dittmer, J.; Strauss, H.-G.; Thomssen, C.; Hauptmann, S Ezrin expression is related to poor prognosis in FIGO stage I endometrioid carcinomas. Mod. Pathol. 2006, 19, 581-587. [CrossRef]

203. Sulaiman, S.A.; Ab Mutalib, N.-S.; Jamal, R. miR-200c Regulation of Metastases in Ovarian Cancer: Potential Role in Epithelial and Mesenchymal Transition. Front. Pharmacol. 2016, 7. [CrossRef]

204. Natalia, M.A.; Alejandro, G.T.; Virginia, T.J.; Alvarez-Salas, L.M. MARK1 is a Novel Target for miR-125a-5p: Implications for Cell Migration in Cervical Tumor Cells. MicroRNA 2018, 7, 54-61. [CrossRef]

205. Tang, X.; Yang, M.; Wang, Z.; Wu, X.; Wang, D. MicroRNA-23a promotes colorectal cancer cell migration and proliferation by targeting at MARK1. Acta Biochim. Biophys. Sin. 2019, 51, 661-668. [CrossRef]

206. Cheng, Y.; He, C.; Wang, M.; Ma, X.; Mo, F.; Yang, S.; Han, J.; Wei, X. Targeting epigenetic regulators for cancer therapy: Mechanisms and advances in clinical trials. Signal Transduct. Target. Ther. 2019, 4, 62. [CrossRef]

207. Eich, M.-L.; Athar, M.; Ferguson, J.E.; Varambally, S. EZH2-Targeted Therapies in Cancer: Hype or a Reality. Cancer Res. 2020, 80, 5449-5458. [CrossRef]

208. Krill, L.; Deng, W.; Eskander, R.; Mutch, D.; Zweizig, S.; Hoang, B.; Ioffe, O.; Randall, L.; Lankes, H.; Miller, D.S.; et al. Overexpression of enhance of Zeste homolog 2 (EZH2) in endometrial carcinoma: An NRG Oncology/Gynecologic Oncology Group Study. Gynecol. Oncol. 2020, 156, 423-429. [CrossRef]

209. Roh, J.-W.; Choi, J.E.; Han, H.D.; Hu, W.; Matsuo, K.; Nishimura, M.; Lee, J.-S.; Kwon, S.Y.; Cho, C.H.; Kim, J.; et al. Clinical and biological significance of EZH2 expression in endometrial cancer. Cancer Biol. Ther. 2020, 21, 147-156. [CrossRef]

210. Hou, X.; Zhao, M.; Wang, T.; Zhang, G. Upregulation of estrogen receptor mediates migration, invasion and proliferation of endometrial carcinoma cells by regulating the PI3K/AKT/mTOR pathway. Oncol. Rep. 2014, 31, 1175-1182. [CrossRef] [PubMed]

211. Guo, R.X.; Wei, L.H.; Tu, Z.; Sun, P.M.; Wang, J.L.; Zhao, D.; Li, X.P.; Tang, J.M. 17 beta-estradiol activates PI3K/Akt signaling pathway by estrogen receptor (ER)-dependent and ER-independent mechanisms in endometrial cancer cells. J. Steroid Biochem. Mol. Biol. 2006, 99, 9-18. [CrossRef] [PubMed]

212. Petrie, W.K.; Dennis, M.K.; Hu, C.; Dai, D.; Arterburn, J.B.; Smith, H.O.; Hathaway, H.J.; Prossnitz, E.R. G Protein-Coupled Estrogen Receptor-Selective Ligands Modulate Endometrial Tumor Growth. Obstet. Gynecol. Int. 2013, 2013, 472720. [CrossRef] [PubMed]

213. Zhou, J.; Song, T.; Gong, S.; Zhong, M.; Su, G. microRNA regulation of the expression of the estrogen receptor in endometrial cancer. Mol. Med. Rep. 2010, 3, 387-392. [CrossRef] [PubMed]

214. Gao, Q.; Ye, F.; Xia, X.; Xing, H.; Lu, Y.; Zhou, J.; Ma, D. Correlation between PTEN expression and PI3K/Akt signal pathway in endometrial carcinoma. J. Huazhong Univ. Sci. Technol. Med. Sci. Hua Zhong Ke Ji Da Xue Xue Bao Yi Xue Ying De Wen Ban Huazhong Keji Daxue Xuebao. Yixue Yingdewen Ban 2009, 29, 59-63. [CrossRef]

215. Xu, X.; Lai, Y.; Hua, Z.C. Apoptosis and apoptotic body: Disease message and therapeutic target potentials. Biosci. Rep. 2019, 39. [CrossRef]

216. Carneiro, B.A.; El-Deiry, W.S. Targeting apoptosis in cancer therapy. Nat. Rev. Clin. Oncol. 2020, 17, 395-417. [CrossRef]

217. Lin, J.; Song, T.; Li, C.; Mao, W. GSK-3 $\beta$ in DNA repair, apoptosis, and resistance of chemotherapy, radiotherapy of cancer. Biochim. Biophys. Acta. Mol. Cell Res. 2020, 1867, 118659. [CrossRef]

218. Luo, S.; Rubinsztein, D.C. BCL2L11/BIM: A novel molecular link between autophagy and apoptosis. Autophagy 2013, 9, 104-105. [CrossRef]

219. Mohr, A.M.; Mott, J.L. Overview of microRNA biology. Semin. Liver Dis. 2015, 35, 3-11. [CrossRef]

220. Owen, K.L.; Brockwell, N.K.; Parker, B.S. JAK-STAT Signaling: A Double-Edged Sword of Immune Regulation and Cancer Progression. Cancers 2019, 11, 2002. [CrossRef]

221. Subramaniam, K.S.; Omar, I.S.; Kwong, S.C.; Mohamed, Z.; Woo, Y.L.; Mat Adenan, N.A.; Chung, I. Cancer-associated fibroblasts promote endometrial cancer growth via activation of interleukin-6/STAT-3/c-Myc pathway. Am. J. Cancer Res. 2016, 6, 200-213. 
222. Janiszewska, M.; Primi, M.C.; Izard, T. Cell adhesion in cancer: Beyond the migration of single cells. J. Biol. Chem. 2020, 295, 2495-2505. [CrossRef]

223. Rutherford, E.J.; Hill, A.D.K.; Hopkins, A.M. Adhesion in Physiological, Benign and Malignant Proliferative States of the Endometrium: Microenvironment and the Clinical Big Picture. Cells 2018, 7, 43. [CrossRef]

224. Schäfer, M.K.E.; Altevogt, P. L1CAM malfunction in the nervous system and human carcinomas. Cell. Mol. Life Sci. 2010, 67, 2425-2437. [CrossRef]

225. Giordano, M.; Cavallaro, U. Different Shades of L1CAM in the Pathophysiology of Cancer Stem Cells. J. Clin. Med. 2020, 9 , 1502. [CrossRef]

226. Zeimet, A.G.; Reimer, D.; Huszar, M.; Winterhoff, B.; Puistola, U.; Azim, S.A.; Müller-Holzner, E.; Ben-Arie, A.; van Kempen, L.C.; Petru, E.; et al. L1CAM in early-stage type I endometrial cancer: Results of a large multicenter evaluation. J. Natl. Cancer Inst. 2013, 105, 1142-1150. [CrossRef]

227. Kommoss, F.K.F.; Karnezis, A.N.; Kommoss, F.; Talhouk, A.; Taran, F.-A.; Staebler, A.; Gilks, C.B.; Huntsman, D.G.; Krämer, B.; Brucker, S.Y.; et al. L1CAM further stratifies endometrial carcinoma patients with no specific molecular risk profile. Br. J. Cancer 2018, 119, 480-486. [CrossRef]

228. Dellinger, T.H.; Smith, D.D.; Ouyang, C.; Warden, C.D.; Williams, J.C.; Han, E.S. L1CAM is an independent predictor of poor survival in endometrial cancer-An analysis of The Cancer Genome Atlas (TCGA). Gynecol. Oncol. 2016, 141, 336-340. [CrossRef]

229. Berger, A.A.; Dao, F.; Levine, D.A. Angiogenesis in endometrial carcinoma: Therapies and biomarkers, current options, and future perspectives. Gynecol. Oncol. 2021, 160, 844-850. [CrossRef]

230. Rubinstein, M.M.; Dickinson, S.; Narayan, P.; Zhou, Q.; Iasonos, A.; Ma, W.; Lakhman, Y.; Makker, V. Bevacizumab in advanced endometrial cancer. Gynecol. Oncol. 2021. [CrossRef]

231. Cheng, Y.; Gao, X.H.; Li, X.J.; Cao, Q.H.; Zhao, D.D.; Zhou, J.R.; Wu, H.X.; Wang, Y.; You, L.J.; Yang, H.B.; et al. Depression promotes prostate cancer invasion and metastasis via a sympathetic-cAMP-FAK signaling pathway. Oncogene 2018, 37, $2953-2966$. [CrossRef]

232. Jiang, K.; Yao, G.; Hu, L.; Yan, Y.; Liu, J.; Shi, J.; Chang, Y.; Zhang, Y.; Liang, D.; Shen, D.; et al. MOB2 suppresses GBM cell migration and invasion via regulation of FAK/Akt and cAMP/PKA signaling. Cell Death Dis. 2020, 11, 230. [CrossRef]

233. Zhang, H.; Kong, Q.; Wang, J.; Jiang, Y.; Hua, H. Complex roles of cAMP-PKA-CREB signaling in cancer. Exp. Hematol. Oncol. 2020, 9, 32. [CrossRef]

234. Levine, D.A.; Getz, G.; Gabriel, S.B.; Cibulskis, K.; Lander, E.; Sivachenko, A.; Sougnez, C.; Lawrence, M.; Kandoth, C.; Dooling, D.; et al. Integrated genomic characterization of endometrial carcinoma. Nature 2013, 497, 67-73. [CrossRef]

235. Coll-de la Rubia, E.; Martinez-Garcia, E.; Dittmar, G.; Gil-Moreno, A.; Cabrera, S.; Colas, E. Prognostic Biomarkers in Endometrial Cancer: A Systematic Review and Meta-Analysis. J. Clin. Med. 2020, 9, 1900. [CrossRef]

236. Hutt, S.; Tailor, A.; Ellis, P.; Michael, A.; Butler-Manuel, S.; Chatterjee, J. The role of biomarkers in endometrial cancer and hyperplasia: A literature review. Acta Oncol. 2019, 58, 342-352. [CrossRef]

237. Xie, Y.; Ma, X.; Chen, L.; Li, H.; Gu, L.; Gao, Y.; Zhang, Y.; Li, X.; Fan, Y.; Chen, J.; et al. MicroRNAs with prognostic significance in bladder cancer: A systematic review and meta-analysis. Sci. Rep. 2017, 7, 5619. [CrossRef]

238. Parol, M.; Gzil, A.; Bodnar, M.; Grzanka, D. Systematic review and meta-analysis of the prognostic significance of microRNAs related to metastatic and EMT process among prostate cancer patients. J. Transl. Med. 2021, 19, 28. [CrossRef]

239. Ferreira, P.; Roela, R.A.; Lopez, R.V.M.; Del Pilar Estevez-Diz, M. The prognostic role of microRNA in epithelial ovarian cancer: A systematic review of literature with an overall survival meta-analysis. Oncotarget 2020, 11, 1085. [CrossRef] [PubMed]

240. Zografos, E.; Zagouri, F.; Kalapanida, D.; Zakopoulou, R.; Kyriazoglou, A.; Apostolidou, K.; Gazouli, M.; Dimopoulos, M.-A. Prognostic role of microRNAs in breast cancer: A systematic review. Oncotarget 2019, 10, 7156-7178. [CrossRef] [PubMed]

241. Hong, D.S.; Kang, Y.K.; Borad, M.; Sachdev, J.; Ejadi, S.; Lim, H.Y.; Brenner, A.J.; Park, K.; Lee, J.L.; Kim, T.Y.; et al. Phase 1 study of MRX34, a liposomal miR-34a mimic, in patients with advanced solid tumours. Br. J. Cancer 2020, 122, 1630-1637. [CrossRef] [PubMed]

242. Beg, M.S.; Brenner, A.J.; Sachdev, J.; Borad, M.; Kang, Y.K.; Stoudemire, J.; Smith, S.; Bader, A.G.; Kim, S.; Hong, D.S. Phase I study of MRX34, a liposomal miR-34a mimic, administered twice weekly in patients with advanced solid tumors. Investig. New Drugs 2017, 35, 180-188. [CrossRef] [PubMed]

243. Forterre, A.; Komuro, H.; Aminova, S.; Harada, M. A Comprehensive Review of Cancer MicroRNA Therapeutic Delivery Strategies. Cancers 2020, 12, 1852. [CrossRef]

244. Van Zandwijk, N.; Pavlakis, N.; Kao, S.C.; Linton, A.; Boyer, M.J.; Clarke, S.; Huynh, Y.; Chrzanowska, A.; Fulham, M.J.; Bailey, D.L.; et al. Safety and activity of microRNA-loaded minicells in patients with recurrent malignant pleural mesothelioma: A first-in-man, phase 1, open-label, dose-escalation study. Lancet Oncol. 2017, 18, 1386-1396. [CrossRef]

245. Callegari, E.; Elamin, B.K.; Giannone, F.; Milazzo, M.; Altavilla, G.; Fornari, F.; Giacomelli, L.; D’Abundo, L.; Ferracin, M.; Bassi, C.; et al. Liver tumorigenicity promoted by microRNA-221 in a mouse transgenic model. Hepatology 2012, 56, 1025-1033. [CrossRef]

246. Kalinkova, L.; Kajo, K.; Karhanek, M.; Wachsmannova, L.; Suran, P.; Zmetakova, I.; Fridrichova, I. Discriminating miRNA Profiles between Endometrioid Well- and Poorly-Differentiated Tumours and Endometrioid and Serous Subtypes of Endometrial Cancers. Int. J. Mol. Sci. 2020, 21, 6071. [CrossRef]

247. Fridrichova, I.; Kalinkova, L.; Karhanek, M.; Smolkova, B.; Machalekova, K.; Wachsmannova, L.; Nikolaieva, N.; Kajo, K. miR-497-5p Decreased Expression Associated with High-Risk Endometrial Cancer. Int. J. Mol. Sci. 2020, 22, 127. [CrossRef] 